\title{
Solitary waves in the excitable Burridge-Knopoff model
}

\author{
J.E. Morales, G. James, A. Tonnelier \\ INRIA Grenoble - Rhône-Alpes, Bipop Team-Project, Inovallée, 655 Avenue de l'Europe, \\ 38334 Saint Ismier Cedex, France.
}

\begin{abstract}
The Burridge-Knopoff model is a lattice differential equation describing a chain of blocks connected by springs and pulled over a surface. This model was originally introduced to investigate nonlinear effects arising in the dynamics of earthquake faults. One of the main ingredients of the model is a nonlinear velocity-dependent friction force between the blocks and the fixed surface. For some classes of non-monotonic friction forces, the system displays a large response to perturbations above a threshold, which is characteristic of excitable dynamics. Using extensive numerical simulations, we show that this response corresponds to the propagation of a solitary wave for a broad range of friction laws (smooth or nonsmooth) and parameter values. These solitary waves develop shock-like profiles at large coupling (a phenomenon connected with the existence of weak solutions in a formal continuum limit) and propagation failure occurs at low coupling. We introduce a simplified piecewise linear friction law (reminiscent of the McKean nonlinearity for excitable cells) which allows us to obtain an analytical expression of solitary waves and study some of their qualitative properties, such as wave speed and propagation failure. We propose a possible physical realization of this system as a chain of impulsively forced mechanical oscillators. In certain parameter regimes, non-monotonic friction forces can also give rise to bistability between the ground state and limit-cycle oscillations and allow for the propagation of fronts connecting these two stable states.
\end{abstract}

Keywords: Solitary waves, Burridge-Knopoff model, Excitable media, Lattice dynamical systems.

\section{Introduction}

A significant body of work has been devoted to elucidating nonlinear mechanisms of earthquakes [1-3]. Almost fifty years ago, Burridge and Knopoff [4] introduced a nonlinear lattice model to investigate the generation of earthquakes along faults, or more generally the occurrence of dynamical instabilities at frictional interfaces. The Burridge-Knopoff (BK) model formally describes a chain of blocks connected by springs and pulled over a surface, each block being attached to a spring pulled at constant velocity and subject to a friction force 
with the surface. When considering two plates under friction, the blocks can either correspond to a discretization of a plate or account for an existing microstructure [5]. It has been shown that this simple slider-block model is able to reproduce some statistical features of earthquakes generated by fault dynamics, see [6-8] and references therein.

A key feature of the BK model lies in the friction force between the blocks and the fixed surface, which depends nonlinearly, often non-monotonically, on sliding velocity. Non-monotone behaviours of the average kinetic friction can be inferred from microscopic models of sliding surfaces, in which interactions between asperities or atoms are incorporated through a local periodic potential $[9,10]$ (see also [11] for more references on the modelling of friction at small scales). Along the same line, experiments performed at the macroscale with a broad range of materials have revealed that the steady-state kinetic friction coefficient is non-monotone versus sliding velocity (see [12] for a review). Friction is velocity-strengthening (i.e. frictional resistance increases with sliding velocity) for high enough velocities; a phenomenon which can originate from radiation of waves within the bulk $[4,10]$, frictional heating [13], or hydrodynamic viscous friction for interfaces containing fluids [14] (see [12] for additional mechanisms). Conversely, friction becomes velocity-weakening in a regime of lower velocities $[12,13,15]$; a behaviour intimately linked with the occurrence of stick-slip instabilities and earthquake phenomena $[2,14]$ (additional fundamental mechanisms involved in such instabilities are reviewed in [16]).

Different types of friction laws have been introduced in order to model such velocity-strengthening or weakening regimes at the macroscale and describe stick-slip phenomena. Rate-and-state laws incorporating state variables for the frictional interface are frequently used, see $[2,13,17,18]$ for reviews. Detailed bifurcation studies have been performed for the BK model with a single block and several rate-and-state laws (see e.g. [17, 19] and references therein), and quantitative agreement with experimental data on Hopf bifurcations have been reported [20]. For chains of blocks, BK models with rate-and-state friction laws have been investigated in $[18,21,22]$ through numerical simulations, but their mathematical analysis remains a delicate task. Indeed, the study of large amplitude excitations in such models is made more difficult by the additional degrees of freedom introduced to describe the state of the interface (but small amplitude waves are simpler to address, see section 2 of [23]). Another type of friction law has been developed to account for the weakening of fault gouges by acoustic fluidization [24], a phenomenon which can be at the origin of slip instabilities [25]. This higher-dimensional model involves a nonlinear diffusion equation for the elastic energy density in the fault core. In particular, analysing the different regimes of steady-state friction requires to solve a one-dimensional nonlinear boundary value problem and study the associated stationary bifurcations [24].

A simpler class of widely used friction laws is given by generalized Coulomb laws with velocity-dependent kinetic friction coefficient [26]. In that case, the set-valued character of the friction laws requires an adapted numerical treatment [27-29] and can lead to analytical complications in dynamical studies (see [23], section 1.4). Alternatively, single-valued laws can be used to approximate 
set-valued friction laws [6] or account for additional physical effects leading to velocity-strengthening at small enough velocities, such as internal dissipation induced by shear deformations [4] or adhesive friction [30, 31].

The dynamics of the BK model has been extensively studied in the case of steady-state velocity-weakening friction. Depending on the choice of parameters and system size, this regime can lead to chaotic dynamics or to the propagation of nonlinear wavetrains [18, 32-34]. In particular, periodic travelling waves close to solitary waves (with highly localized slipping events propagating at constant velocity) have been reported in numerical and analytical studies $[18,23,33,35$, $36]$.

In this paper, we consider a different situation corresponding to 'spinodal' friction laws $[4,17,31,37]$, where steady-state friction is velocity-strengthening both for small and large enough velocities and an intermediate velocity-weakening region exists. This situation has been reported in a number of friction experiments performed with rocks, rubber and hydrogels [31, 38, 39]. In that case, the dynamics of a single block can be described by a Van der Pol type equation, a situation frequently encountered in the modelling of excitable media [40-43]. Numerical studies of the BK model with spinodal friction laws (either generalized Coulomb laws or regularizations thereof) have revealed different types of wave patterns, ranging from synchronous oscillations, periodic travelling waves and phase fronts $[44,45]$ to chimera-like states (see Fig. 8 of reference [46] for an early observation of this phenomenon). These different studies were focused on the oscillatory regime where the pulling velocity lies within the velocityweakening region.

With regard to spatially localized travelling waves, the existence of fronts has been established in a continuum limit of the BK model [47] (see also [4850] for numerical studies of rupture fronts in other continuum models based on rate-and-state laws). However, the existence of localized waves was not established so far for the spatially discrete BK model with spinodal friction laws. The analysis of finite amplitude travelling waves in the discrete system is more delicate because it leads to a nonlinear advance-delay differential equation for the wave profiles. In order to tackle this problem, an interesting inverse approach was described in reference [51], where particular friction laws were computed in order to fit prescribed explicit wave profiles. An analytical moving kink solution (with block displacements given by an odd function of the moving frame coordinate $z$ ) was proposed but turns out to be erroneous (the velocity dependent friction force induced by the kink is even in $z$, and cannot be balanced by the odd inertial and stress terms in the dynamical equation (10) of [51]).

In the present paper, we analyse in detail the existence of localized waves in the discrete BK model with spinodal friction laws, using both extensive numerical simulations and analytical techniques. We restrict our attention to solitary waves and treat the case of fronts in a companion paper [52]. We consider the excitable regime where the pulling velocity lies within a velocity-strengthening domain of the spinodal friction laws (above velocity-weakening). In that case, each single block admits a stable state of continuous slip but displays a large response to perturbations above some threshold. When blocks become coupled, 
our numerical simulations reveal that this response generates a solitary wave for a broad range of friction laws (smooth or nonsmooth) and parameter values. In the case of regularized Coulomb laws, the solitary wave consists of a moving localized region where sticking occurs, in contrast to the propagation of localized slipping events previously reported for velocity-weakening friction [33].

We analyse in detail the influence of parameters on the existence and qualitative properties of solitary waves. For low enough coupling between blocks, we observe a phenomenon of propagation failure corresponding to the rapid extinction of initially propagating pulses. We find that solitary waves develop shocklike profiles in the opposite limit of large coupling, a phenomenon connected with the existence of weak solutions in a formal continuum limit. When the pulling velocity is decreased towards the boundary of the velocity-strengthening domain, the dynamics of the blocks becomes underdamped and we observe solitary waves with oscillatory tails, while propagation failure takes place above some critical pulling velocity. For certain friction laws (near the transition to a velocity-weakening law), we also observe bistability between continuous slip and limit-cycle oscillations and the existence of propagating fronts connecting these two stable states.

In order to obtain analytical expressions for solitary waves and explain some of their qualitative properties, we introduce a simplified piecewise linear friction law with two velocity-strengthening regions separated by a (negative) jump discontinuity. The discontinuity in the friction force can be interpreted as a rough approximation for the existence of a small intermediate velocity-weakening region. Alternatively, we propose a possible physical realization of this piecewise linear model as a chain of impulsively forced mechanical oscillators (stiffness and damping are assumed linear). In this system, excitability arises from an impulsive force applied in the direction of motion above some critical deflection. Such types of piecewise linear nonlinearities have been extensively used for the mathematical study of travelling waves in different types of PDEs [53-56] and spatially discrete systems [57-68]. Following this approach allows us to obtain explicit solitary waves in the form of oscillatory integrals and numerically compute their profiles and wave speed. We explain the occurrence of propagation failure below some critical coupling by the existence of a saddle-node bifurcation of solitary waves.

It is worthwhile to stress that we obtain fully localized solitary waves, i.e. blocks lie in the state of stable slip at infinity on both sides of the chain (this constitutes another important difference with the previous works $[23,33]$ ). Similar solutions have been previously obtained for other types of excitable lattice dynamical systems where the coupling is diffusive rather than elastic. These systems correspond to spatially discrete FitzHugh-Nagumo equations with either smooth [69] or piecewise linear [61] bistable nonlinearities. In our case, the properties of solitary waves are quite different at large coupling with the occurrence of shocks in the BK model.

The outline of this paper is as follows. In section 2, we introduce the excitable BK model and different types of spinodal friction laws. In section 3, we use numerical simulations to study the existence and shape of solitary waves 


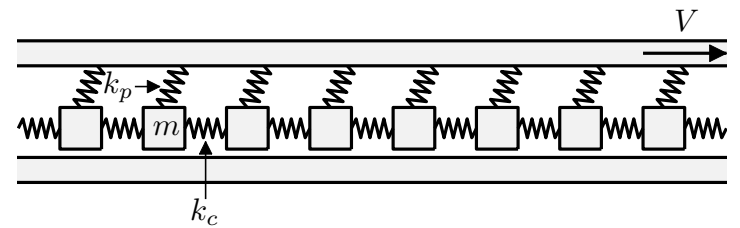

Fig. 1 - Mechanical interpretation of the Burridge-Knopoff model.

depending on parameters. We also consider a continuum limit of the model and illustrate the bistable dynamics near the transition to a velocity-weakening law. Section 4 provides analytical results for the idealized piecewise linear friction law. Section 5 summarizes the main findings and points out interesting open problems. The chain of impulsively forced mechanical oscillators leading to the piecewise linear excitable BK model is described in Appendix A.

\section{The excitable Burridge-Knopoff model}

\subsection{Dynamical equations and solitary waves}

The BK model describes the interaction of two solids in contact, one being pulled at a constant speed $V$ and discretized as a chain of blocks of mass $m$ (see Fig. 1). The blocks are connected to nearest-neighbours through Hookean springs of stiffness $k_{c}$ and to the upper plate by springs of stiffness $k_{p}$. Each block is subject to a nonlinear friction force with the lower surface, with a kinetic friction coefficient depending on the sliding velocity.

The motion of an isolated block is given by

$$
m \frac{d^{2} x}{d t^{2}}+k_{p} x+f_{0} F\left(\frac{V+\frac{d x}{d t}}{v_{1}}\right)=0
$$

where $x(t)$ is the displacement of the block relative to the point of attachment on the moving plate. The friction force is described by the nonlinear function $F$ and involves two scaling parameters $f_{0}$ and $v_{1}$.

Using the change of variable $y(\bar{t})=x(t) / \ell$ with $\ell=f_{0} / k_{p}, \bar{t}=t v_{1} / \ell$ and introducing the nondimensional parameters $\gamma=m v_{1}^{2} k_{p} / f_{0}^{2}, \bar{V}=V / v_{1}$, Eq. (1) can be reformulated as the dimensionless dynamical system on the plane:

$$
\begin{aligned}
\frac{d y}{d t} & =u, \\
\gamma \frac{d u}{d t} & =-F(V+u)-y,
\end{aligned}
$$

where the rescaled time $\bar{t}$ and pulling velocity $\bar{V}$ have been rewritten as $t$ and $V$.

The qualitative dynamics of (2) highly depends on the shape of the nonlinear function $F(v)$. In this paper, we consider spinodal friction laws similar to the 
A

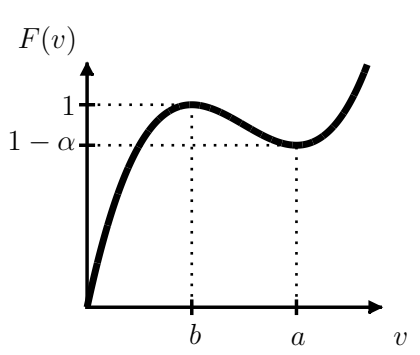

B

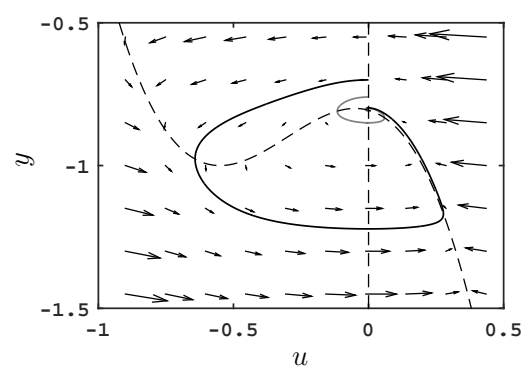

D
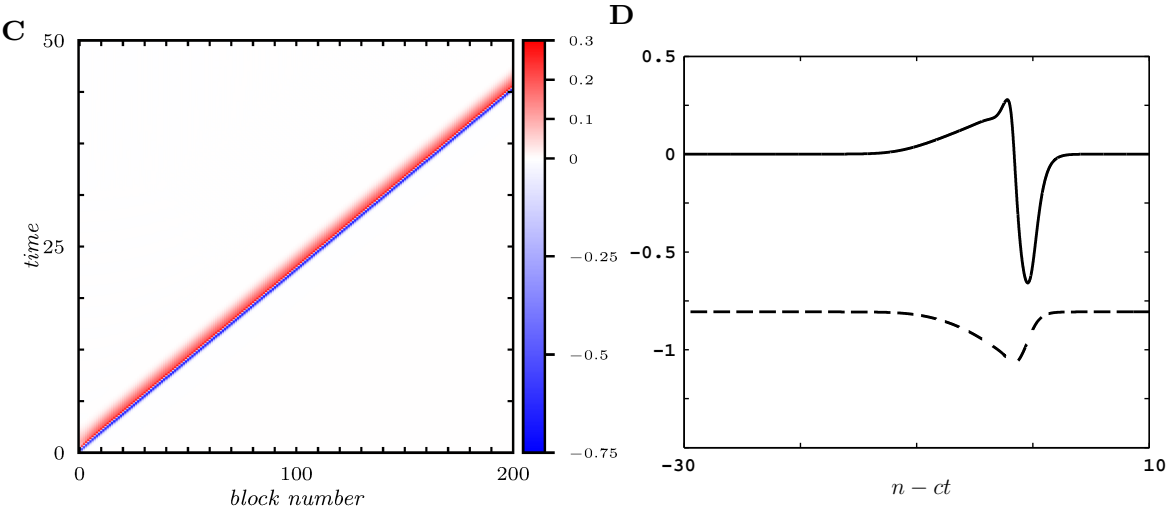

Fig. 2 - A Spinodal friction force defined by the cubic polynomial $F_{c}(v)=3.2 v^{3}-7.2 v^{2}+4.8 v$ for $v>0$. In $\mathbf{B}$, the responses of an isolated block to perturbations of different magnitudes are shown in the phase plane (the arrows represent the vector field (2) and dashed lines correspond to nullclines). For a strong enough perturbation of the resting state, a large trajectory is elicited (black line), whereas a small perturbation does not produce a significant response (grey line). Parameters are $V=1.05$ and $\gamma=0.15$. Panels $\mathbf{C}, \mathbf{D}$ illustrate the response of the system when blocks are coupled (we fix $k=3$ ). A sufficiently strong initial perturbation of the ground state at one end of the chain generates a solitary wave (we set $\left.\left(u_{0}(0), y_{0}(0)\right)=\left(u_{s}-1, y_{s}\right)\right)$. Panel $\mathbf{C}$ provides a space-time diagram of block velocities. In $\mathbf{D}$, velocities (full line) and displacements (dashed line) are plotted in the co-moving frame. 
one plotted in Fig. 2A, with $F$ decreasing on an interval $[b, a]$ and increasing on $[0, b] \cup[a,+\infty)$.

Equation (2) admits a unique equilibrium $\left(u_{s}, y_{s}\right)=(0,-F(V))$ corresponding to continuous slip. For $V \in(b, a)$, the steady state is unstable (the condition $F^{\prime}(V)<0$ corresponds to a negative damping) and the system displays an oscillatory behaviour $[44,45]$. If the friction law is smooth (i.e. $F$ is $C^{1}$ ), the existence of a periodic orbit of (2) has been proved under mild conditions on $F$, see e.g. [70] (Theorem 1.2, p. 176). The uniqueness of a stable periodic orbit can be established under additional conditions of $F$ [70] (Theorem 4.6, p. 229). When $F$ is $C^{3}$, periodic solutions appear through Hopf bifurcations at $V=b$ and $V=a$ (the bifurcation at $V=a, b$ is supercritical for $F^{(3)}(V)>0$ and subcritical if $F^{(3)}(V)<0$, see section 1.3 of [23]). For less regular spinodal friction laws the available results are scarcer. However, for $F$ piecewise linear with three zones (i.e. $F$ linear on $(-\infty, b),(b, a)$ and $(a,+\infty))$ and continuous, the existence of a unique stable periodic orbit has been shown in [71] for $V \in(b, a)$.

In this paper, we focus on the different regime $V>a$ where the steady state is stable and equation (2) displays excitability. For these parameter values (and similarly for $V \in[0, b]$ ), perturbing the steady state above some threshold produces a large trajectory in the phase plane, whereas small perturbations are immediately damped, see Fig. 2B. In addition, there exists a refractory period where the block is unresponsive to further perturbations.

From now on, we address the dynamics of a chain of blocks in the excitable regime $V>a$. In the classical BK model, the force acting on the $n$-th block due to nearest-neighbours is $k_{c} \Delta_{d} x_{n}$, where $x_{n}$ corresponds to block positions and $\Delta_{d} x_{n}=x_{n+1}-2 x_{n}+x_{n-1}$ denotes the discrete Laplacian. Performing the same rescaling as for (2) and introducing the dimensionless coupling parameter $k=k_{c} / k_{p}$, the BK model can be recast in the form

$$
\begin{aligned}
\frac{d y_{n}}{d t} & =u_{n}, \\
\gamma \frac{d u_{n}}{d t} & =k \Delta_{d} y_{n}-F\left(V+u_{n}\right)-y_{n},
\end{aligned}
$$

where $n \in \mathbb{Z}$.

In this work, we report results on the existence of solitary wave solutions where each block returns to the rest state after wave passage. More precisely, solitary wave solutions of (3) take the form

$$
\begin{aligned}
& u_{n}(t)=\varphi(n-c t), \\
& y_{n}(t)=\psi(n-c t),
\end{aligned}
$$

with $(\varphi( \pm \infty), \psi( \pm \infty))=(0,-F(V))$. The function $(\varphi, \psi)$ defines the waveform and $c$ denotes the wave speed. Typical position and velocity waveforms are illustrated by Fig. 2D for a cubic friction law. Conditions for the excitation of solitary waves will be studied in detail in section 3.1, where we analyse under which conditions the perturbation of one block propagates to its neighbours. In an appropriate parameter range (for $V>a$ not too large and $k$ not too small), 
we find that perturbations of the rest state lying above some threshold quickly evolve into a solitary wave (see the spatiotemporal plot of Fig. 2C). Once the regime of steady propagation is reached, the solitary wave profile and velocity are independent of the magnitude of the initial perturbation. For perturbations with small enough amplitude, solitary waves fail to propagate and we observe a convergence towards the spatially uniform rest state.

In the next section, we introduce the general class of spinodal friction laws for which we shall numerically establish the existence of solitary waves in the BK model.

\subsection{Friction laws}

Several types of non-monotonic friction laws have been proposed in the literature. Many studies consider generalized Coulomb laws with a multivalued part at the origin (see Fig. 3A,B). These friction laws involve a sticking condition when a block achieves a zero velocity with respect to the lower plate. Single-valued regularized Coulomb laws are also often considered for numerical purpose, or to account for the existence of a velocity-strengthening region for low sliding velocities. In addition, friction laws that switch from a velocityweakening to a velocity-strengthening regime for large sliding velocities are also relevant for applications, as discussed in section 1. Combining these different effects results in spinodal friction laws of the type depicted in Fig. 2A and Fig. 3D-E.

In this paper, we investigate the dynamics of the BK model using the prototypical smooth spinodal friction law given by the cubic function $F_{c}(v)=$ $3.2 v^{3}-7.2 v^{2}+4.8 v$, depicted in Fig. $2 \mathrm{~A}$. We shall denote by $v=a$ the local minimum of the spinodal friction force, the above cubic friction law corresponding to $a=1$. In order to analyse the effects of a larger class of spinodal friction laws (in particular with different degrees of smoothness or stiffness), we also introduce the following class of piecewise linear functions

$$
F_{P L}(v ; a, b, \mu)= \begin{cases}\frac{v}{b}, & 0 \leq v<b, \\ \alpha\left(\frac{v-b}{b-a}\right)+1, & b<v<a, \\ \mu(v-a)+1-\alpha, & a<v,\end{cases}
$$

that have been normalized so that $F_{P L}(b)=1$ (this is equivalent to choosing $f_{0}$ as the maximal friction force in (1)). For $v<0$ we set $F_{P L}(v)=-F_{P L}(-v)$. The parameter $b$ is the threshold where the transition from velocity-strengthening to velocity-weakening occurs. If $\mu \geq 0$, velocity strengthening is recovered above the sliding velocity $a$ and $F(a)=1-\alpha(\alpha \in(0,1))$ corresponds to a local minimum of the friction force. Our computations will be performed for $a=1$, which is equivalent to choosing the scaling parameter $v_{1}$ as the local minimum of the friction force in (1).

We shall consider more specifically four piecewise linear functions defined as follows and depicted in Fig. 3C-F. A crude approximation of spinodal friction 


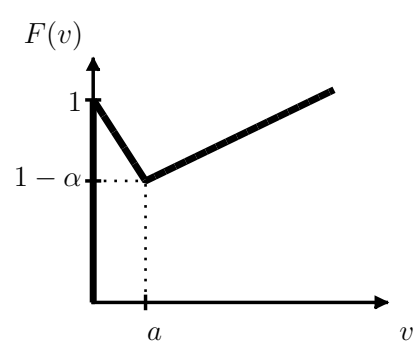

A

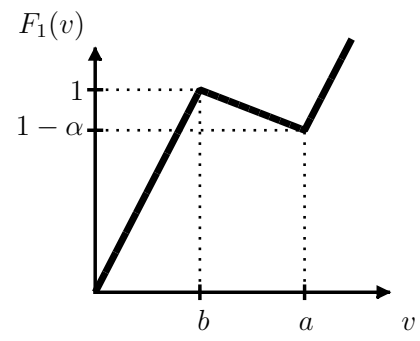

D

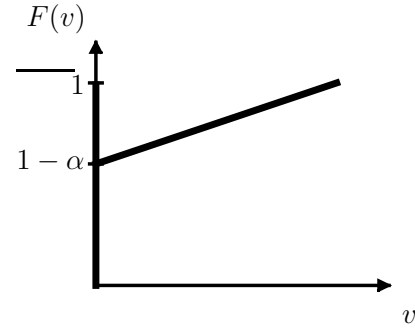

B

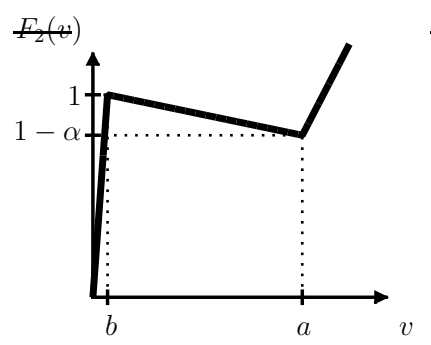

$\mathbf{E}$

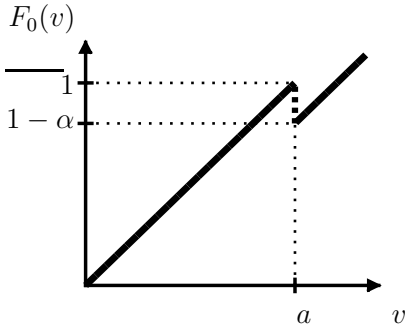

C

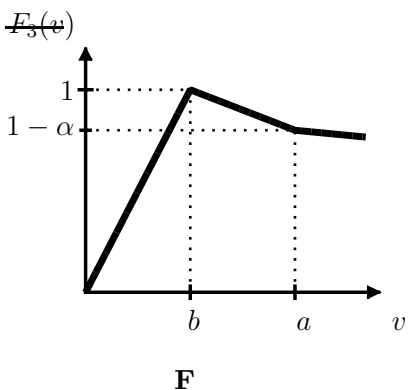

Fig. 3 - Typical piecewise linear friction laws for the Burridge-Knopoff model. A-E display different spinodal friction laws (velocity-weakening for an intermediate range of sliding velocities) whereas $\mathbf{F}$ is velocity-weakening for large enough velocities. $\mathbf{A}, \mathbf{B}$ correspond to multivalued laws (generalized Coulomb laws). Velocity-weakening is assumed instantaneous in cases $\mathbf{B}, \mathbf{C}$ (the velocity-weakening domains are reduced to a jump discontinuity in the friction force). The single-valued laws $\mathbf{C}-\mathbf{F}$ are used in the present work, with $a=1, \alpha=0.2$, $b=0.5$ in cases $\mathbf{D}, \mathbf{F}$ and $b=0.01$ in case $\mathbf{E}$.

laws is given by $F_{0}(v)=F_{P L}(v ; a, a, 1 / a)$ (Fig. 3C). This friction function can be rewritten as

$$
F_{0}(v)=\frac{v}{a}-\alpha H(v-a), \quad v>0
$$

where $H$ is the Heaviside step function. With this friction law, the damping ratio in the two velocity-strengthening regimes are assumed identical and the velocity-weakening region is reduced to a jump discontinuity. The ensuing discontinuity of the vector field of (2) across the line $u=a-V<0$ induces a strict excitability threshold, unlike the smooth excitation threshold arising from continuous friction laws (and illustrated in Fig. 2B for the cubic friction law). Interestingly, for the piecewise linear and discontinuous law $F_{0}$, the BK model can be also interpreted as a chain of impulsively forced mechanical oscillators (see Appendix A).

The friction law defined by $F_{1}=F_{P L}(v ; a, a / 2,2 / a)$ is obtained from $F_{0}$ with the addition of an intermediate velocity-weakening region with finite thickness (Fig. 3D). To regularize a multivalued generalized Coulomb law, we also consider $F_{2}(v)=F_{P L}(v ; a, b, 2 / a)$ with a small value of $b$ (Fig. 3E). In that case the friction law is close to the multivalued case depicted in Fig. 3A. It would be also interesting to investigate the limit case of Fig. 3B (considered e.g. in [47]), 
but the numerical simulation of the BK model is more delicate in that case and will be investigated in future work. Finally, to investigate the transition from a velocity-strengthening law to a velocity-weakening law at large sliding velocities we introduce $F_{3}(v)=F_{P L}(v ; a, a / 2, \mu)$ (Fig. $\left.3 \mathrm{~F}\right)$ where the slope $\mu$ may be negative.

\section{Qualitative properties of solitary waves}

\subsection{Regimes of solitary wave generation}

In this section, the existence of solitary waves is explored for system (3) with free-end boundary conditions and the spinodal friction laws introduced in section 2.2. We consider the cubic law $F_{c}$ and the piecewise linear laws $F_{0}, F_{1}$ and $F_{2}$ (the values of parameters $a, b$ and $\alpha$ are the same as in Fig. 3). All the numerical results are illustrated with the fixed mass parameter $\gamma=0.15$, which leads to an overdamped regime for the piecewise linear friction laws considered herein (the underdamped case will be briefly discussed at the end of this section).

We investigate the excitable regime with the pulling velocity $V$ satisfying $a<V<V_{\max }$, where $V_{\max }>a$ is defined through $F\left(V_{\max }\right)=1$. The two limiting regimes, $V \rightarrow a^{+}$or $V \rightarrow V_{\max }^{-}$, will be referred to as the regimes of small or maximal pulling velocity, respectively. To explore intermediate velocity regimes, we also consider a medium pulling velocity $V_{\text {med }}>a$ defined through $F\left(V_{\text {med }}\right)=1-\alpha / 2$.

Propagating waves are initiated using a shock-like initial condition: a sufficiently strong perturbation is applied on the resting state $\left(u_{s}, y_{s}\right)_{n}$ for a subset of blocks in the network, possibly reduced to a single block near the left edge of the chain. The initial perturbation is applied to the position or to the velocity. For the numerical integration, the adaptive scheme Lsoda [72] is employed with a minimal error tolerance set at $1.5 \cdot 10^{-8}$. A time step $\Delta t=0.001$ is used to follow the numerical solution. We recall that the nondimensional time $t$ corresponds to units of $f_{0} /\left(k_{p} v_{1}\right)$, where the velocity $v_{1}$ is the local minimum of the unscaled spinodal friction force in (1).

Unless stated otherwise, simulations are done with a network size and for a time duration such that a stationary solution is reached. For ensuring a stable localized wave propagation, typical network size is of order one hundred for small pulling velocities, whereas medium velocities require around ten thousands blocks. Such large network simulations have to be performed because the increase of $V$ also requires increasing $k$ in order to excite solitary waves (see below). This parameter regime results in higher wave speed, convergence time towards steady state, and pulse width (simulations for these network sizes can be efficiently addressed using Python programming language).

For $a<V<V_{\max }$, localized solutions can propagate stably along the network for sufficiently large values of the coupling constant $k$ (typical wave profiles are plotted in Fig. 4 and 5). We observe that the wave speed and width increase with the coupling value when the other parameters are fixed, while increasing $\gamma$ 
decreases the wave speed. The magnitude of the initial perturbation required to generate a propagation depends on $V$. In the regime of small pulling velocity, a velocity perturbation of magnitude in the interval $[0.125,2.5]$ is sufficient to excite a solitary wave for all friction forces. An excitation of the position is more efficient to produce trains of solitary waves and a perturbation magnitude in the interval $[0.01,0.5]$ is used (this higher sensitivity to perturbations of positions is clearly illustrated for a single block by Fig. 2-B). For a medium pulling velocity $\left(V \sim V_{\text {med }}\right.$ ), a stronger perturbation on the velocity is needed, up to 10 , whereas perturbations in the position remain similar.

For $V>V_{\max }$ or if $k$ lies below a ( $V$-dependent) critical value $k^{*}$, we observe that localized excitations fail to produce travelling waves. This phenomenon is well documented for excitable lattices of diffusive type and has been coined propagation failure [73]. As $V$ increases, the critical value $k^{*}$ increases and a vertical asymptote is observed near $V=V_{\max }$. This is shown in Fig. $4 \mathrm{~B}$ where the regime of existence and stability of solitary waves is indicated in the $V-k$ plane for the cubic friction law. Qualitatively similar results are obtained for the other piecewise linear spinodal laws (results not shown). Some values of $k^{*}$ are provided in Table 1 for different friction laws and pulling velocities. The increase of $k^{*}$ versus $V$ can be intuitively explained by the geometry of excitability of a single block: for small pulling velocities, the initial state of the network is near the right knee of the spinodal friction function and a small perturbation is sufficient to reach the threshold of excitability. As $V$ increases, the steady state gets away from the threshold separatrix and the perturbation has to be stronger in order to initiate a large response of the block. Above $V_{\max }$ the excitability property of the block disappears.

\begin{tabular}{ccl}
\hline Friction law & $\begin{array}{c}\text { Small pulling velocity } \\
V=1.025\end{array}$ & \multicolumn{1}{c}{ Medium pulling velocity } \\
& $V_{\text {med }}$ \\
\hline$F_{c}(v)$ & $k^{*} \approx 1.4$ & $k^{*} \approx 360.8$ \\
$F_{0}(v)$ & $k^{*} \approx 0.43$ & $k^{*} \approx 1915.3$ \\
$F_{1}(v)$ & $k^{*} \approx 4.5$ & $k^{*} \approx 99$ \\
$F_{2}(v)$ & $k^{*} \approx 48$ & $k^{*} \approx 602$ \\
\hline
\end{tabular}

Table 1 - Critical coupling $k^{*}$ for different friction laws and pulling velocities.

As it can be inferred from Fig. 4 and 5, the waveforms obtained for different friction laws, pulling velocities $V$ and coupling strengths $k$ share common features. The displacement profile presents a hump whereas the velocity profile shows a rapid downstroke followed by an upstroke, a property related to the fact that $\int_{\mathbb{R}} u_{n}(t) d t=y_{n}(+\infty)-y_{n}(-\infty)=0$. Interestingly, the shape of typical velocity profiles is reminiscent of the action potential of spiking neurons. The discontinuous law defined by $F_{0}$ produces a rather symmetric velocity profile where the positive and negative peaks are quasi-equidistant from the resting state. This property is more pronounced in the regime of small pulling velocity and tends to break down as $V$ increases. The symmetry is broken for the other friction laws $\left(F_{c}, F_{1}, F_{2}\right.$ and $\left.F_{3}\right)$ with the existence of a velocity-weakening 


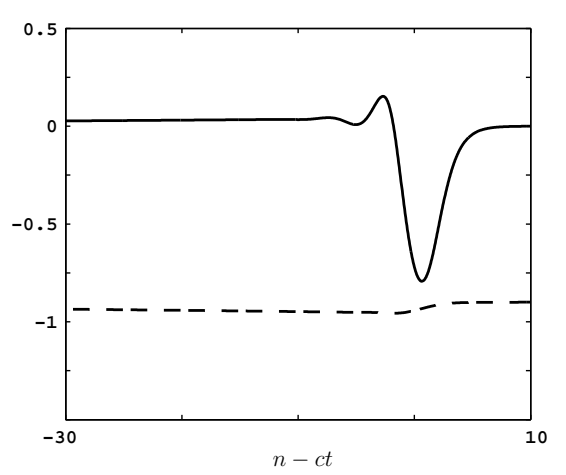

A

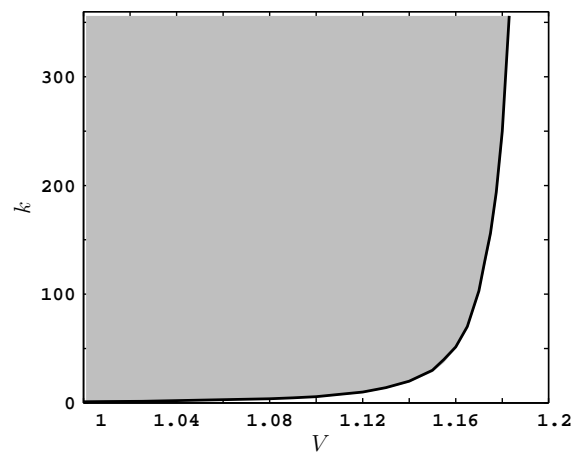

B

Fig. 4 - A Wave profiles of the velocity $\varphi(n-c t)$ (solid) and the displacement $\psi(n-c t)$ (dashed) for the cubic friction law $F_{c}(v), V=1.183$ and $k=400$. The wave speed of the localized travelling wave is $c \approx 50.05$. B Regimes of existence and stability of solitary waves in the $V-k$ plane (grey shading) for the cubic friction law. The solid line indicates the critical coupling values $k^{*}$ below which propagation failure occurs. Regimes are computed from numerical simulations in which the wave is initiated by imprinting a velocity $u_{p}$ to the first block in the chain (magnitude up to $u_{p}=-10$ ). Note that here $V_{\max }=1.25$.

region. The positive peak of the velocity profile is less marked and the magnitude of the negative spike is determined by the distance between the stable steady state and the left branch of the velocity-nullcline (see Fig. 2B). When the velocity-weakening range increases, the amplitude of the negative peak of the velocity profile becomes larger while the wave speed decreases.

The tail of the velocity profile presents either a monotonic or an oscillatory return to the resting state. This feature is determined by the modes around the resting state. Linearization of (3) around the steady state $\left(u_{n}, y_{n}\right)=(0,-F(V))$ reads

$$
\gamma \ddot{y}_{n}=k \Delta y_{n}-y_{n}-F^{\prime}(V) \dot{y}_{n} .
$$

In order to analyse the relaxation towards the rest state, we look for normal modes $y_{n}(t)=A e^{i q n+\sigma(q) t}+c . c$. where $A \neq 0$ and $\sigma(q)$ are two complex numbers, $q \in[0, \pi]$ and c.c. stands for complex conjugate. This leads to

$$
\sigma(q)=\left[-F^{\prime}(V) \pm \sqrt{\Delta(q)}\right] \frac{1}{2 \gamma},
$$

where $\Delta(q)=F^{\prime}(V)^{2}-4 \gamma\left[1+4 k \sin ^{2}(q / 2)\right]$ is a decreasing function of $q$ on $[0, \pi]$. The asymptotic decay rate is controlled by the slowest decaying mode (obtained for $q=0$ ), which also describes the relaxation of the uncoupled system. When $V$ approaches the local minimum of the cubic friction force $F_{c}$, the value of $F_{c}^{\prime}(V)$ tends towards 0 and all modes become underdamped ( $\sigma$ being imaginary). The resulting feature is an oscillatory tail of the wave as shown in Fig. 5D where the envelope of the oscillations becomes larger as $V$ decreases. Due to the constant derivative of the piecewise linear friction forces $F_{0}, F_{1}, F_{2}$ 


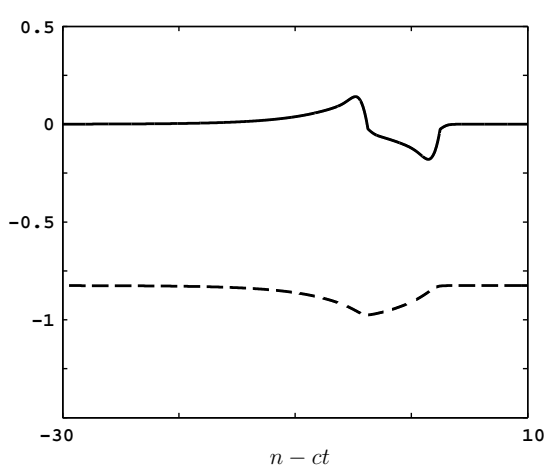

A

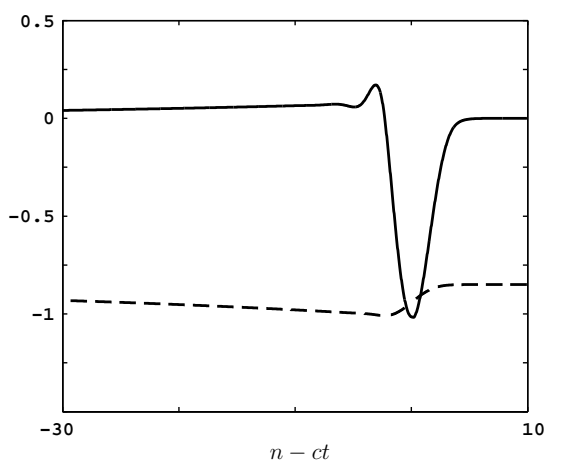

C

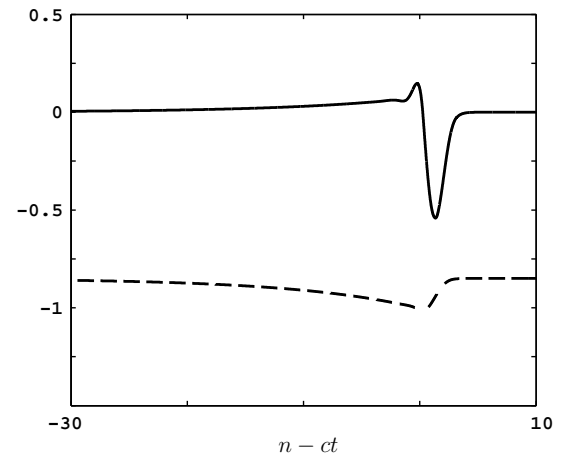

B

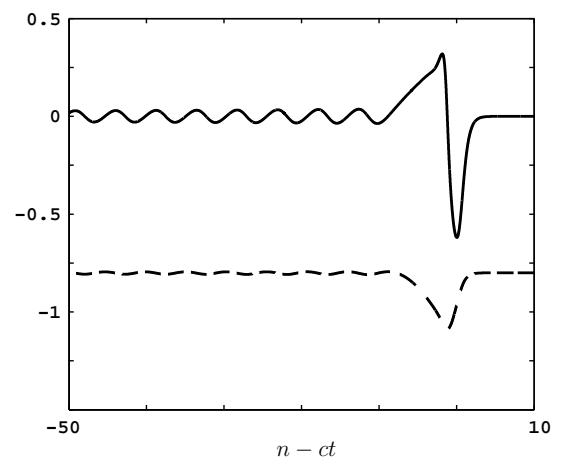

D

Fig. 5 - Plot of the waveforms $\varphi(n-c t)$ (solid) and $\psi(n-c t)$ (dashed) for, $\mathbf{A} F_{0}(v), V=1.025$, $k=2, \mathbf{B} F_{1}(v), V=1.025, k=5, \mathbf{C} F_{2}(v), V=1.025, k=70$ and, $\mathbf{D} F_{c}(v), V=1, k=2$. The local minimum of each spinodal friction force is located at $a=1$. The computed wave speed values are $c=4.39, c=5.36, c=21.23, c=4.04$ for A-D, respectively. Simulations are done for a strong perturbation $\left(u_{p}=-10\right)$ of the velocity of the first block.

for sliding velocities above $a$, the modes do not depends on $V$. In that case the occurrence of oscillatory behaviour can be analysed as above and only depends on $\gamma, k$ and the slope $\mu$ in (5).

In the above study, we have restricted our attention to linear nearest-neighbours coupling. It is worthwhile to note that similar types of solitary waves can exist in the case of nonlinear interactions between blocks. This is illustrated in Fig. 6 for Hertzian interactions and a linear contact law.

In the following two subsections, we explore two limiting regimes of particular interest in the BK model : the continuum limit and a bistability regime.

\subsection{Continuum limit}

We are interested in the study of solitary waves when the discrete BK model approaches a continuum limit, i.e. for $k \gg 1$. Near the continuum limit, system 

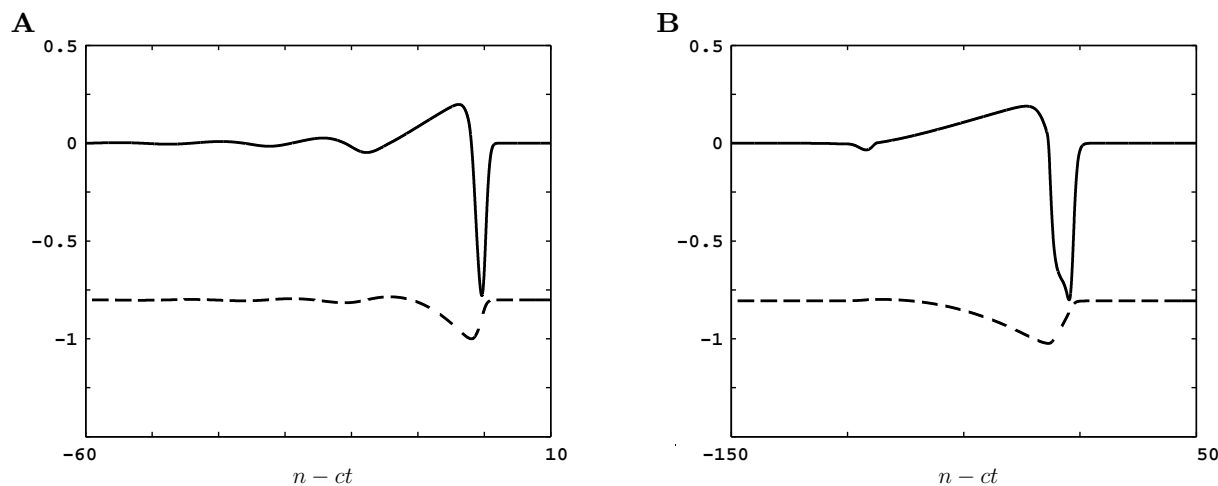

Fig. 6 - Solitary waves profiles $\varphi(n-c t)=u_{n}(t)($ solid $)$ and $\psi(n-c t)=y_{n}(t)($ dashed $)$ obtained with (A) Hertzian and (B) linear contact interactions in the BK model with cubic friction law $F_{c}$. The Hertzian coupling is obtained by replacing the discrete linear Laplacian in (3) by $k\left[\left(y_{n-1}-y_{n}\right)_{+}^{3 / 2}-\left(y_{n}-y_{n+1}\right)_{+}^{3 / 2}\right]$, and the linear contact law corresponds to $k\left[\left(y_{n-1}-y_{n}\right)_{+}-\left(y_{n}-y_{n+1}\right)_{+}\right]$, where $(x)_{+}=\max (0, x)$. Parameters are: $\mathbf{A}, k=20$, $V=1.025$ and $\mathbf{B}, k=150, V=1.05$. The wave speeds are $\mathbf{A}, c \approx 6.36$ and $\mathbf{B}, c \approx 32.47$, respectively. The wave is generated from the chain at rest except for the first block, where the initial velocity and position are $\mathbf{A}\left(u_{s}-2, y_{s}\right)$ and $\mathbf{B}\left(u_{s}-1, y_{s}\right)$.

(3) takes the form:

$$
\gamma \frac{\partial^{2} y}{\partial t^{2}}+F\left(V+\frac{\partial y}{\partial t}\right)+y=\frac{\partial^{2} y}{\partial x^{2}}+\frac{h^{2}}{12} \frac{\partial^{4} y}{\partial x^{4}}+\mathcal{O}\left(h^{4}\right)
$$

where we have set $y_{n}(t)=y(x, t), x=n / \sqrt{k}$ and $h=1 / \sqrt{k} \ll 1$. The equation obtained by neglecting $\mathcal{O}\left(h^{4}\right)$ terms in (9) is known as a Boussinesq-type approximation. We look for solitary wave solutions of (9) and set $y(x, t)=Y(s)$ with $s=x / \tilde{c}-t$ (for system (3), this corresponds to solitary waves with large velocity $c=\tilde{c} \sqrt{k}$ ). The above Ansatz leads to the ODE

$$
\Lambda Y^{\prime \prime}-F\left(V-Y^{\prime}\right)-Y=-\frac{h^{2}}{12 \tilde{c}^{4}} Y^{(4)}+\mathcal{O}\left(h^{4}\right)
$$

where $\Lambda=1 / \tilde{c}^{2}-\gamma$.

The fourth derivative at the right side of (10) may be useful to account for the appearance of high frequency oscillations (see below), but this problem will not be analysed in the present study. In this work we restrict to the second order model obtained by neglecting $\mathcal{O}\left(h^{2}\right)$ terms, which reads

$$
\Lambda Y^{\prime \prime}(s)-F\left(V-Y^{\prime}(s)\right)-Y=0 .
$$

This equation admits the unique equilibrium $\left(Y, Y^{\prime}\right)=(-F(V), 0)$. In what follows we study the case of the cubic friction law $F=F_{c}$. The equilibrium point is a saddle point for $\Lambda>0$, an unstable point (source) for $\Lambda<0$ and $\Delta \geq 0$, an unstable focus for $\Lambda<0$ and $\Delta<0$, where $\Delta=F^{\prime}(V)^{2}+4 \Lambda$. The existence of a smooth solitary wave corresponds to the existence of an homoclinic 
orbit for equation (11). In the unstable configuration $(\Lambda<0)$ it is clear that such an orbit cannot exist. In the saddle case $(\Lambda>0)$, an additional equilibrium inside the homoclinic orbit has to be present because the phase space is twodimensional. Since there is only one single equilibrium point, smooth solitary waves cannot exist in the continuum model (11).

However for large $k$ values, numerical simulations of the discrete BK model reveal the existence of solitary waves. Typical profiles of these waves are shown in Fig. 7. The velocity component displays two shocks (resulting in two slope discontinuities for the displacement component), and we observe long-lived (i.e. metastable) oscillations in the vicinity of the rear shock. These oscillations are not strictly periodic and display a modulation within the shock (in Fig. $7 \mathrm{~B}$, the time between two velocity maxima decreases from $7.5 \cdot 10^{-3}$ to 4.8 . $10^{-3}$ across the shock thickness). An interesting open question concerns the existence of exact travelling wave solutions of this type (i.e. with an oscillatory shock) in the BK model (3) or in the higher order continuum model obtained by neglecting $\mathcal{O}\left(h^{4}\right)$ terms in (10). These problems will be addressed in future work using numerical continuation techniques. After a very long transient, the fast oscillations near the rear shock disappear, which leads to the shock-wave profiles displayed in the right column of Fig. 7. Given the long time scale of simulations, it is not clear whether this slow drift towards a non-oscillatory shock is a dynamical property of the BK model or is due to numerical integration errors.

The profile of the asymptotic non-oscillatory pulse can be analytically captured by considering equation (11) with $\Lambda=0$, which corresponds to fixing $\tilde{c}=\sqrt{1 / \gamma}$. In that case, the solitary wave of (3) propagates at the sound velocity $c=\sqrt{k / \gamma}$. This case leads to the differential-algebraic equation

$$
Y=-F\left(V-Y^{\prime}\right) .
$$

Solutions of (12) are plotted in Fig. 7 (blue lines). They correspond to weak solutions $y(x, t)=Y(x / \tilde{c}-t)$ of the nonlinear PDE obtained by setting $h=0$ in (9). In particular, the solution of (12) shown the right column of Fig. 7 perfectly matches the stationary profiles shown in Fig. 7C,E. For the parameter values provided in Fig. 7, the wave speed of the numerically observed solitary wave is $c \approx 2587$ and the analytical approximation $c=\sqrt{k / \gamma}=2582$ is quite accurate (relative error $<2 \cdot 10^{-3}$ ). In the case of oscillatory shocks, solutions of (12) provide reasonably accurate approximations of block displacements (see Fig. 7D), but they only approximate velocity oscillations on average near the rear shock (Fig. 7B).

\subsection{Bistability regime}

The excitable nature of system (3) can give rise to an oscillatory behaviour, similar to the self-sustained oscillations produced by excitable membranes in biology [74]. The state of continuous slip loses its stability when the friction law operates a transition from a velocity-strengthening regime to a velocity-

weakening regime. For the friction law $F_{3}$ defined in section 2.2, this case occurs 


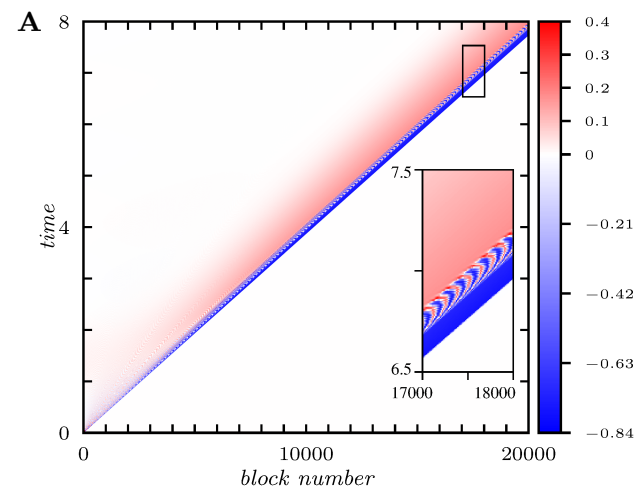

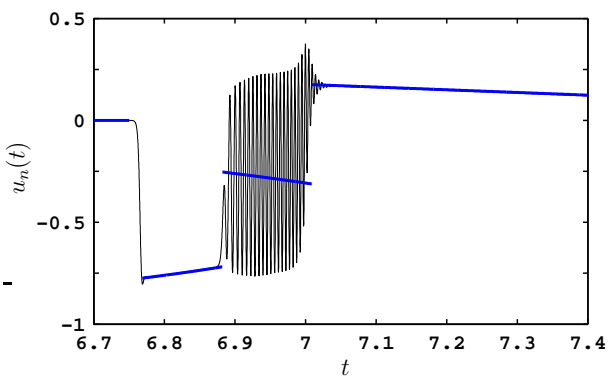

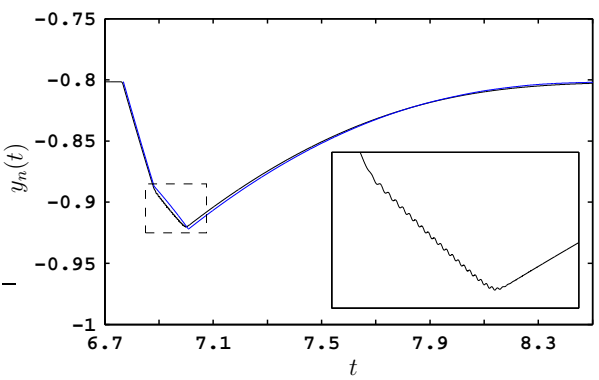

$\mathbf{F}$

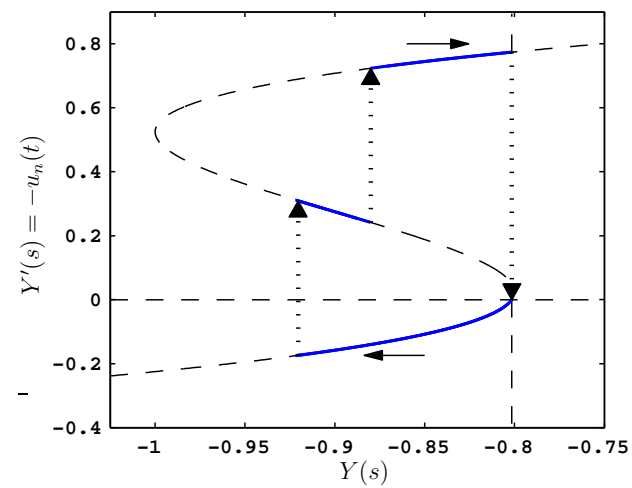

C

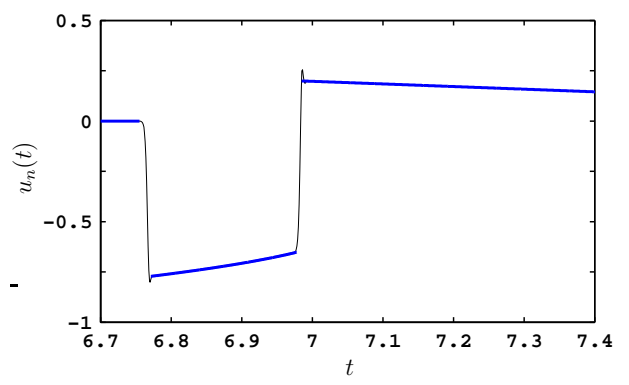

$\mathbf{E}$

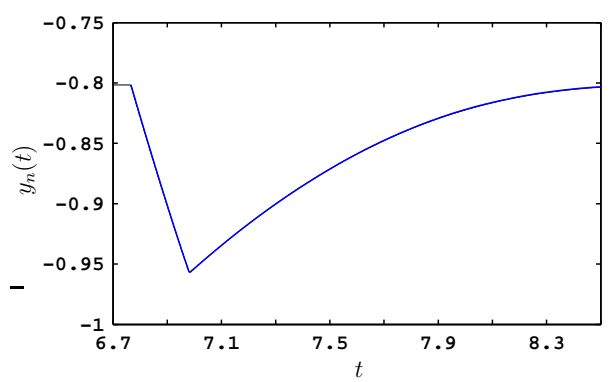

G

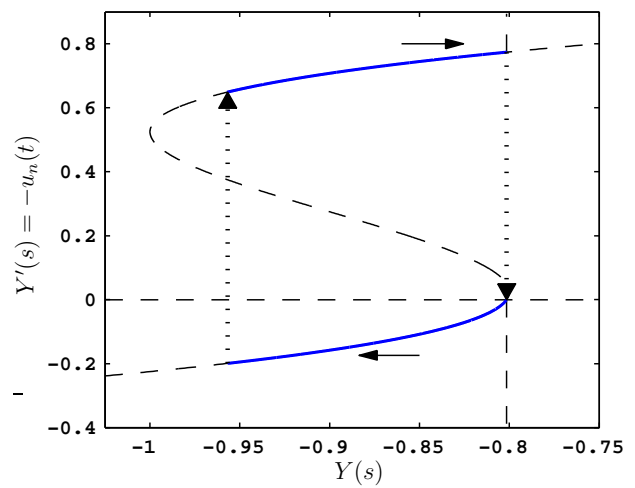

Fig. 7 - Simulation of the BK model (3) in the high coupling limit $\left(k=10^{6}\right)$, for $V=1.025$ and friction law $F_{c}(v)$. A Spatiotemporal plot of $u_{n}(t)$. When the pulse reaches $n>15000$, a quasistationary state is reached. B Plot of $u_{n}(t)$ (black line) for $n=17500$ and $\mathbf{D}$, plot of $y_{n}(t)$ (black line) where the inset is an enlargement that shows the small fluctuations of $y_{n}(t)$ in the highly oscillatory region of $u_{n}(t)$. In $\mathbf{C}$ and $\mathbf{E}$, we plot the asymptotic solitary wave profiles for $n>95000$ (we have shifted the time origin in these two figures, so that figures B-C and D-E are easier to compare). In $\mathbf{F}$ and $\mathbf{G}$, the blue lines are different solutions of (12) that match the quasi-stationary waveforms shown in $\mathbf{B}, \mathbf{C}$ and $\mathbf{D}, \mathbf{E}$, respectively. Nullclines of (11) are represented in the $\left(Y, Y^{\prime}\right)$ plane as dashed lines. Solid arrows indicate the time evolution. Dotted arrows represent the fast transition between the blue lines of plots $\mathbf{B}$ and $\mathbf{C}$. Initial excitation: $\left(u_{0}(0), y_{0}(0)\right)=\left(u_{s}-10, y_{s}\right)$. 
when $V>a$ and the slope parameter $\mu$ switches from positive to negative. The dynamics of a single block is illustrated in the top panels of Fig. 8. In the velocity-weakening regime $\mu<0$, there exists a large-amplitude stable limit cycle, which persists in the range $\mu>0$ provided $\mu$ is not too large (see the bifurcation diagram in Fig. 8A). For the state of continuous slip, a subcritical Hopf bifurcation occurs at $\mu=0$, leading to the existence of an unstable periodic orbit when $\mu$ is positive and not too large. If $\mu$ further increases, the two periodic orbits disappear through a saddle-node bifurcation. Consequently, there exists a narrow interval of positive values of $\mu$ where the model exhibits bistability between the state of continuous slip and a limit cycle (a typical phase portrait is shown in Fig. 8B). The two attractors are separated by an unstable periodic orbit, and a change of position (or velocity) can switch the system from rest to oscillation and vice versa.

In this parameter regime, it is an interesting problem to examine the possible spatiotemporal patterns which can be generated by coupling the bistable units. The stability of steady sliding can be analysed through the linear system (7), with eigenvalues given by (8) for $F^{\prime}(V)=\mu$. For $\mu>0$, the equilibrium state $\left(u_{s}, y_{s}\right)$ is locally asymptotically stable, while for $\mu<0$ it becomes unstable. For $\mu>0$, different types of dynamics can be observed depending on the initial perturbation, as exemplified by Fig. 8C-D. In the example of Fig. 8C, a velocity perturbation of one block with large magnitude triggers the propagation of a front with modulated periodic oscillations in the back. While periodic travelling waves are clearly visible at the front edge, in-phase oscillations are taking hold at the beginning of the chain. As shown in Fig. 8B, these oscillations are close to the stable periodic solution studied in Fig. 8A. Figure 8D corresponds to a much smaller perturbation producing complex oscillatory patterns. The dynamics of the BK model in the bistable regime is likely to be very rich and needs to be explored in more detail in future work.

\section{Construction of solitary waves for the discontinuous piecewise lin- ear friction force}

The analysis of exact solitary waves in the BK model leads us to consider an advance-delay differential equation. More precisely, substitution of (4) into (3) yields

$$
\begin{aligned}
-c \psi^{\prime}(\xi) & =\varphi(\xi) \\
-c \gamma \varphi^{\prime}(\xi) & =k[\psi(\xi+1)-2 \psi(\xi)+\psi(\xi-1)]-F(V+\varphi(\xi))-\psi(\xi)
\end{aligned}
$$

where $\xi=n-c t \in \mathbb{R}$ represents the travelling wave coordinate and the wave speed $c$ has to be determined. By differentiating (14) and using (13), one obtains the equation

$$
c^{2} \gamma \frac{d^{2} \varphi}{d \xi^{2}}=k \Delta_{d} \varphi+c \frac{d}{d \xi} F(V+\varphi)-\varphi
$$

supplemented by the boundary conditions $\varphi(\xi) \rightarrow 0$ as $\xi \rightarrow \pm \infty$. 
A

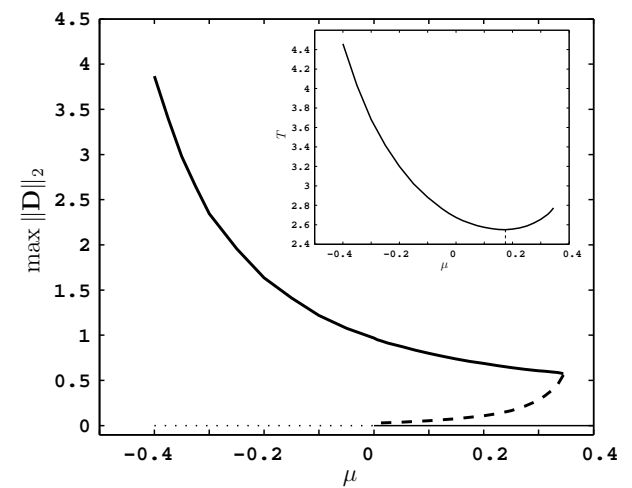

C

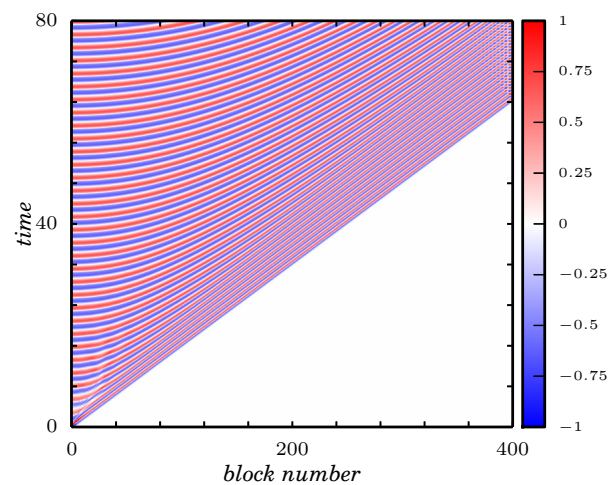

B

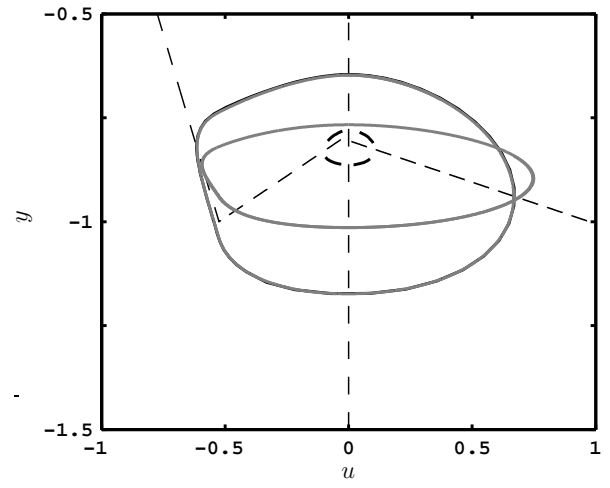

D

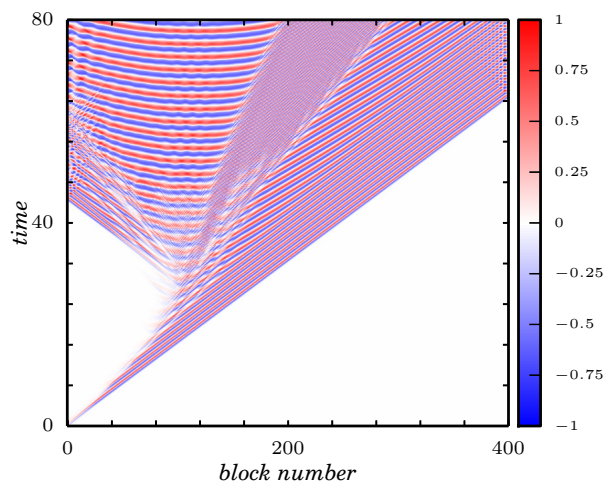

Fig. 8 - Simulations of the BK model with friction law $F_{3}(v)$ in a bistable regime, with $V=1.025$ and $k=5$. A: Bifurcation diagram of a single block that shows the amplitudes of the stable (solid line) and unstable (dashed line) periodic orbits. The amplitude is defined by $\max _{t>0}\|D(t)\|_{2}$, where $D(t)=\left(u(t)-u_{s}, y(t)-y_{s}\right)$ and $\left(u_{s}, y_{s}\right)$ is the equilibrium state. The latter is stable (solid straight line) or unstable (dotted straight line) depending on $\mu$. The inset displays the period $T$ of the stable limit cycle versus $\mu$ (note that $T$ is non-monotone). Panels B,C,D display simulation results for $\mu=0.2$. B: Phase plane of a single block. The nullclines (dashed lines), the stable limit cycle (bold closed curve) and unstable limit cycle (dashed closed curve) are represented. C,D: Wave patterns generated in the chain of blocks. A perturbation $\left(u_{0}(0), y_{0}(0)\right)=\left(u_{s}+u_{p}, y_{s}\right)$ with $u_{p}=-10$ is used in $\mathbf{C}$, whereas $u_{p}=-0.25$ in $\mathbf{D}$. For the simulation shown in $\mathbf{C}$, the oscillations of two blocks $(n=0, n=380)$ are also plotted in figure $\mathbf{B}$ (grey curves), where the trajectory of the first block converges towards the stable limit cycle.

In this section, we study analytically and numerically the localized solutions of (15) for the piecewise linear friction force $F_{0}$ defined in (6), for which the velocity-weakening is assumed to be instantaneous. Numerical simulations presented in section 3.1 suggest that this piecewise linear model captures the main qualitative features of the solitary waves observed for a broader class of friction forces. 
In equation (15), the piecewise linear function $\varphi \mapsto F_{0}(V+\varphi)$ is discontinuous across the threshold $\varphi=-\beta=a-V<0$. This feature leads us to a classification of travelling waves based on the threshold hitting. Let us define the decreasing (finite or infinite) sequence $\left(\xi_{p}\right)_{p}$ through

$$
\varphi\left(\xi_{p}\right)=-\beta .
$$

Translation invariance of the solution allows us to fix $\xi_{0}=0$. Different wave types can be obtained according to their signature $\left(\sigma_{p}\right)_{p}$ defined as (see Fig. 9)

$$
\sigma_{p}=[H(\varphi+\beta)]_{\xi_{p}},
$$

with $H$ denoting the Heaviside step function and $[f]_{\xi_{p}}=f\left(\xi_{p}^{+}\right)-f\left(\xi_{p}^{-}\right)$the jump discontinuity of a function $f$ at $\xi_{p}$. We distinguish between threshold crossing from above, threshold crossing from below, and threshold reaching without crossing that read $\sigma_{p}=-1, \sigma_{p}=1$, and $\sigma_{p}=0$, respectively. The travelling wave solutions are classified according to the changes of sign of $\varphi+\beta$ that is given by the sequence $\left(\sigma_{p}\right)_{p}$. It is worth noting that travelling sliding solutions are excluded, i.e. the solution is not allowed to remain on the discontinuity line of the friction force.

Fixing $\left(\sigma_{p}\right)_{p}$, we have:

$$
\begin{aligned}
\frac{d}{d \xi} F_{0}(V+\varphi) & =\left\{\frac{d}{d \xi} F_{0}(a+\varphi+\beta)\right\}+\sum_{p}\left[F_{0}(a+\varphi+\beta)\right]_{\xi_{p}} \delta_{\xi_{p}} \\
& =\left\{F_{0}^{\prime}(a+\varphi+\beta)\right\}\left\{\varphi^{\prime}\right\}+\left[F_{0}\right]_{a} \sum_{p} \sigma_{p} \delta_{\xi_{p}}, \\
& =\frac{\varphi^{\prime}}{a}-\alpha \sum_{p} \sigma_{p} \delta_{\xi_{p}},
\end{aligned}
$$

where $\delta_{\xi_{p}}$ is the Dirac distribution at $\xi_{p}$. From equation (15) with $F=F_{0}$, travelling waves in the class $\left(\sigma_{p}\right)_{p}$ satisfy the linear non-homogeneous differential equation:

$$
c^{2} \gamma \varphi^{\prime \prime}-\frac{c}{a} \varphi^{\prime}+\varphi=k \Delta_{d} \varphi-c \alpha \sum_{p} \sigma_{p} \delta_{\xi_{p}} .
$$

A solution of (16) defines a solution of (15) if the crossing conditions at $\left(\xi_{p}\right)_{p}$ are satisfied together with the sign conditions given by the signature $\left(\sigma_{p}\right)_{p}$. This leads to the following admissibility conditions

$$
\left\{\begin{array}{l}
\varphi\left(\xi_{2 j}\right)=\varphi\left(\xi_{2 j+1}\right)=-\beta \\
\varphi(\xi)<-\beta \text { if } \xi \in] \xi_{2 j+1}, \xi_{2 j}[ \\
\varphi(\xi)>-\beta \text { otherwise, }
\end{array}\right.
$$

where we assume for clarity that there is no threshold reaching without crossing (no $p$ such that $\sigma_{p}=0$ ).

Applying Fourier transform, we obtain a unique localized solution of (16) given by

$$
\varphi(\xi)=\sum_{p} \int_{\mathbb{R}} e^{i 2 \pi \lambda \xi} g_{c, \xi_{p}}(\lambda) d \lambda
$$




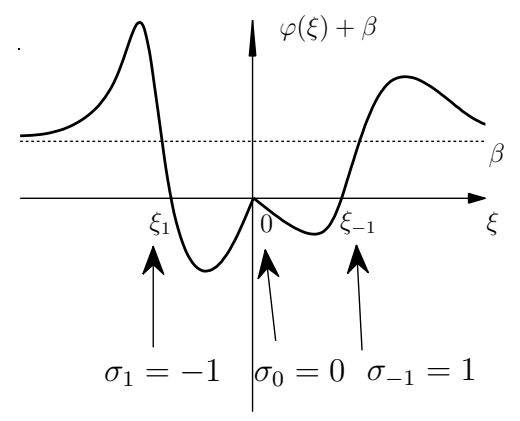

Fig. 9 - Illustration of the signature $\sigma_{p}$ for different threshold crossings.

A

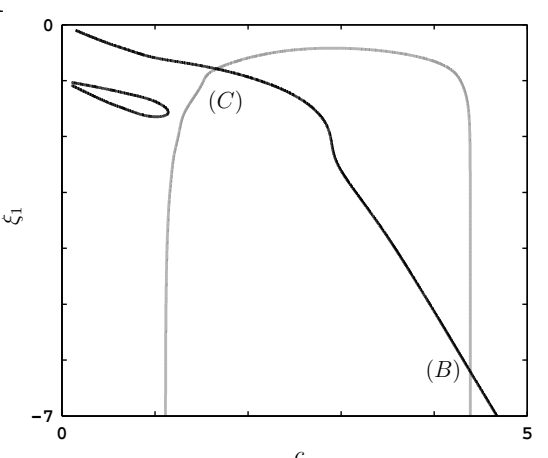

B

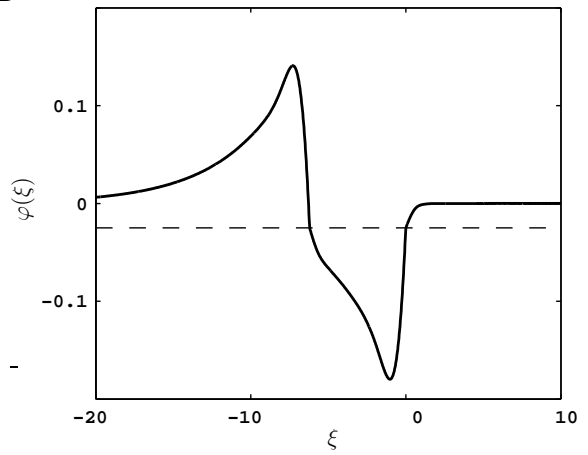

C

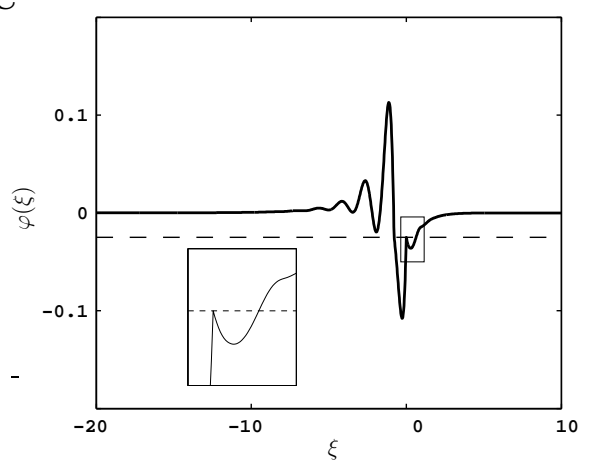

Fig. 10 - In A : plot of the two level curves $\varphi\left(\xi_{1}\right)=-\beta$ (black) and $\varphi(0)=-\beta$ (grey) in the $\left(c, \xi_{1}\right)$ plane. Crossing points provide trial solitary wave solutions through formula (19). The crossing point $(B) \approx(4.39,-6.21)$ is associated with the waveform shown in $\mathbf{B}$ (black line). This analytical solution perfectly matches the solution obtained through dynamical simulation in Fig. 5A (grey dashed line). The crossing point $(C) \approx(1.65,-0.8)$ defines a spurious solution as shown in $\mathbf{C}$, where the enlargement (inset) reveals the violation of the inequality constraints. Parameters are $k=2, V=1.025$. 
where

$$
g_{c, \xi_{p}}(\lambda)=\alpha c \sigma_{p} e^{-2 \pi i \lambda \xi_{p}}\left[4 \pi^{2} c^{2} \lambda^{2} \gamma-4 k \sin ^{2}(\pi \lambda)+\frac{2 \pi i c \lambda}{a}-1\right]^{-1} .
$$

The analytical expression (18) only provides trial solutions. Indeed, the admissibility conditions (17) have to be fulfilled in order to define a solution of (15).

The simplest solitary waves cross the threshold only twice, at $\xi_{0}=0$ and at $\xi_{1}<0$. In that case, (18) simplifies into

$$
\varphi(\xi)=\alpha c \int_{\mathbb{R}} e^{2 \pi i \lambda \xi}\left(1-e^{-2 \pi i \lambda \xi_{1}}\right)\left[4 \pi^{2} c^{2} \lambda^{2} \gamma-4 k \sin ^{2}(\pi \lambda)+\frac{2 \pi i c \lambda}{a}-1\right]^{-1} d \lambda,
$$

and (17) yields two nonlinear equations

$$
\left\{\begin{array}{l}
\varphi(0)=-\beta \\
\varphi\left(\xi_{1}\right)=-\beta
\end{array}\right.
$$

Each equation in system (20) defines a level curve in the plane $\left(c, \xi_{1}\right)$. The crossing points of these two level curves determine the speed $c$ and width $\xi_{1}$ of trial solutions. We numerically compute the level curves using a grid with mesh size 0.001. In order to evaluate (19), we use a Gauss-Kronrod quadrature formula [75] in the truncated interval $\left[-10^{6}, 10^{6}\right]$ (one could alternatively use the method of residues, see e.g. [66]). Approximate solutions of (20) are found with a trust-region dogleg algorithm [76]. As shown in Fig. 10A different crossing points exist.

The inequality constraints of the admissibility conditions (17) have to be fulfilled in order to obtain solutions of (15). A trial solution determined by (19)-(20) which does not satisfy these sign conditions will be denoted as a spurious solution. A plot of the waveform (19) allows one to determine if the sign conditions are fulfilled, see Fig. 10B,C. In this example, one of the two crossing points yields a spurious solution (see the enlargement in Fig. 10C).

In Fig. 11, we plot the $(c, k)$ curve of the solitary wave solutions. The speed curve is U-shaped with a fold at $\left(c^{*}, k^{*}\right)$. For $c>c^{*}$, we obtain a branch of stable solitary waves whose propagation is robust under small random perturbations of the corresponding initial conditions (data not shown). Their profiles and velocities are in perfect agreement with the stable solitary waves generated by exciting a single block (see Fig. 10B and 11). A saddle-node bifurcation of solitary waves occurs at $\left(c^{*}, k^{*}\right)$. Close to this point, there exists a branch of unstable solitary waves with $c<c^{*}$ visible in Fig. 11B. Small initial perturbations of these profiles either result in propagation failure or drive the system towards a stable solitary wave (results not shown). When $k$ increases above a critical value, $\varphi^{\prime}\left(0^{+}\right)$becomes negative and the unstable solitary waves become spurious solutions. This phenomenon corresponds to a border collision bifurcation and is illustrated in the inset of Fig. 10C. In that case, we suspect that a travelling sliding solution appears or an additional threshold crossing occurs (both situations are not captured by the present analysis). 
A

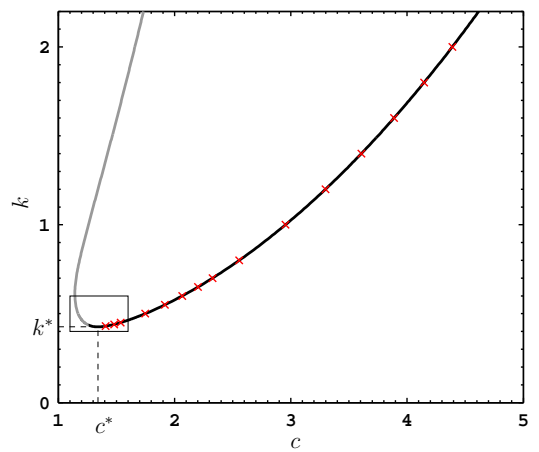

B

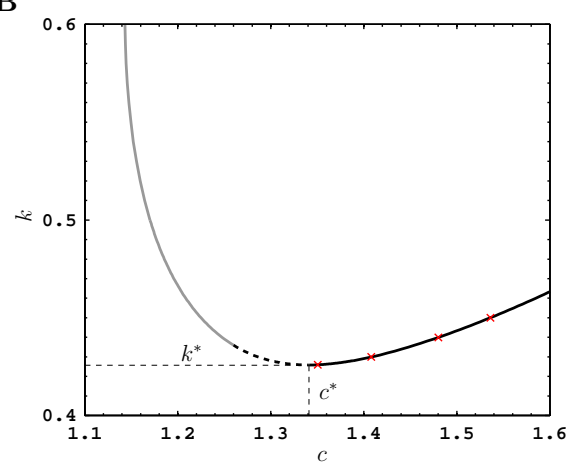

Fig. $11-\mathbf{A}$ : solitary wave speeds $c$ obtained by solving the nonlinear system (20) for different values of the coupling strength $k$ (we have fixed $V=1.025$ ). Panel $\mathbf{B}$ provides a zoom near the fold at $\left(c^{*}, k^{*}\right)\left(k^{*} \approx 0.426\right.$ is the critical coupling below which propagation failure occurs and $c^{*} \approx 1.341$ is the related wave speed). The full line corresponds to stable solitary wave solutions, the dotted line to unstable solitary waves, and the grey line to spurious solutions. The transition from unstable solitary waves to spurious solutions occurs through a border collision bifurcation at $\left(c_{s}, k_{s}\right) \approx(1.260,0.436)$. The red crosses indicate solitary wave velocities obtained from dynamical simulations similar to Fig. $5 \mathrm{~A}$.

\section{Discussion}

In this paper, we have reported the existence of solitary waves in the excitable BK model with linear nearest-neighbours coupling. We have studied how the friction law and parameters influence their formation and qualitative properties. Wave profiles have been analytically obtained for a piecewise linear friction law with a jump discontinuity. Above a critical value of the coupling, we have obtained a branch of stable solitary waves coexisting with unstable waves or spurious solutions, and we have shown that propagation failure at low coupling results from a saddle-node bifurcation. We have proposed in the Appendix a possible mechanical implementation of the BK model with piecewise linear discontinuous nonlinearity, which corresponds to a chain of linear oscillators subjected to impulsive forces applied in the direction of motion when their deflection crosses a specific threshold.

A key problem left open in this paper concerns the theoretical justification of the trial solutions (19)-(20), which requires to prove that the inequality constraints in (17) are satisfied. This problem will be addressed in a next paper in the small coupling limit and for pulling velocities near the jump discontinuity (analytical expansions of the solitary waves can be obtained in that case [61]). In addition, analysing the linear stability of the corresponding solitary waves constitutes another interesting open problem. On another front, it would be interesting to extend our theoretical results to more general friction laws. Different analytical techniques could be applied to bilinear laws regularized by a small spinodal layer, discontinuous laws with two different slopes, the trilinear law (5) or friction laws including a time delay, see e.g. [34, 57, 58, 77-82] and references therein. 
It would be also useful to perform a numerical continuation of solitary waves in the BK model for different types of friction laws and analyse the ensuing bifurcations. In particular, one could determine if the oscillatory shocks observed in dynamical simulations near the continuum limit correspond to exact multibump solitary waves. Alternatively, numerical continuation could be performed at the level of higher-order continuum models. For this purpose, one could resort to the Boussinesq approximation obtained in (9) or other continuum limits involving mixed space-time higher order derivatives [83, 84].

Another future extension of this work will concern multivalued friction laws, i.e. generalized Coulomb laws of spinodal type. In the present work, we have numerically observed solitary wave propagation in the BK model with regularized spinodal Coulomb laws, hence we conjecture the persistence of such waves for multivalued laws.

It would be also relevant to consider generalizations of the BK model involving different types of couplings. We have checked that solitary waves can be also generated in the case of contact interactions (either Hertzian or linear), but a detailed investigation of such models remains to be done, as well as analysing the effect of long-range coupling. Another relevant problem concerns the two-dimensional BK model, where one could search for solitary plane waves $y_{m, n}(t)=\psi(m \cos \theta+n \sin \theta-c t)$ propagating along prescribed directions $e^{i \theta}$ (similarly to the work [60]). In that case, system (13)-(14) would be replaced by an advance-delay differential equation involving two different delays.

In our study of solitary waves, we have only considered the case of a single pulse propagating in an homogeneous chain. It would be also relevant to analyse pulse dynamics near boundaries or defects and to study the interaction between pulses. For example, transitions from pulse annihilation to crossing have been studied in different continuum excitable models such as reactiondiffusion or cross-diffusion systems [56, 85-87]. Considering the BK model, it would be interesting to analyse the possible effects of spatial discreteness and nonsmoothness (in particular for multivalued laws) in such collision processes.

Finally, it would be of interest to experimentally reproduce the solitary waves analysed in the present study. As discussed in Appendix A, MEMS may provide a convenient framework to implement equation (A.1), which is equivalent to the piecewise linear BK model with discontinuous friction law $F_{0}$ introduced in section 2.2. Another possibility would be to consider an electrical equivalent of the BK model consisting of coupled L-C elements and tunnel diodes (see [88] and section 3.2.4 of [89]). Indeed, the tunnel diode has a spinodal current-voltage characteristic similar to the one depicted in Fig. 2A, leading to a negative resistance at intermediate voltage values. This type of system would allow for a great flexibility in the choice of parameters, shaping of nonlinearities and types of coupling among nodes.

The experimental investigation of solitary waves along regular patterned interfaces displaying spinodal frictional resistance would be also of great interest. In this setting, one could tune to some extent the elastic and inertial parameters of the equivalent BK model by selecting appropriate geometries and materials for the asperities and elastic membrane (see e.g. [5] for different configurations). 
In the context of earthquakes, some of the dynamical phenomena occurring in the excitable BK model may be also relevant for the study of creeping faults. Such faults display for long periods of time a stable sliding along with microseismicity, unlike seismic faults that remain predominantly locked between large earthquakes (see [90] for a review). This aseismic sliding is often interpreted in terms of effective velocity-strengthening, but non-monotonic frictional behaviour may occur in certain situations (as reviewed in section 1) and result in an excitable dynamics. In that case, as illustrated by the present study of the simplified BK model, external perturbations lying above some threshold could potentially excite solitary waves along a creeping fault (such perturbations could be typically nucleated at a velocity-weakening portion of the fault, or triggered by the passage of seismic waves radiated from other faults). These solitary waves may be involved in small or large earthquakes or foreshocks, depending on the fault characteristics (in particular its frictional properties). In conclusion, as suggested by the present study, it would be very interesting to simulate more realistic models of creeping faults in order to investigate the existence of excitable regimes and solitary waves, and compare the results to observational data.

\section{Appendix A. Oscillator chain with impulsive excitations}

In this appendix we describe a possible physical implementation of the BK model with piecewise linear and discontinuous friction function $F_{0}$ (Fig. 3C). This system corresponds to a chain of coupled linear oscillators, where each element is subject to an impulsive external force (e.g. a piezoelectric or electrostatic actuation) when reaching a critical deflection $z=\zeta$. The dynamical equations read

$$
m \ddot{z}_{n}+\nu \dot{z}_{n}+k_{p} z_{n}=k_{c} \Delta_{d} z_{n}+\lambda \sum_{\substack{t_{k} \in \mathbb{R}, z_{n}\left(t_{k}\right)=\zeta}} \operatorname{sign}\left(\dot{z}_{n}\left(t_{k}^{-}\right)\right) \delta_{t_{k}},
$$

where $z_{n}(t)$ is the deflection of the $n$th oscillator in the chain. In the right side of (A.1), $\delta_{t_{k}}$ denotes the Dirac distribution at $t=t_{k}$, sign the usual sign function (odd and equal to unity on $(0,+\infty)$ ), and we use the notation $\dot{z}_{n}\left(t_{k}^{-}\right)$(resp. $\left.\dot{z}_{n}\left(t_{k}^{+}\right)\right)$for the left (resp. right) limit of $\dot{z}_{n}$ at $t=t_{k}$. For the series of impulses to be well defined as a distribution, it is assumed that each component $z_{n}(t)$ crosses the critical value $z=\zeta$ for a countable set of times $t_{k}$ (generally depending on $n$ ) without finite accumulation point (i.e. Zeno behaviour does not occur). The external (state-dependent) impulsion has a fixed magnitude $\lambda>0$ and is always applied in the direction of motion. Each oscillator is damped $(\nu>0$ denotes the associated damping constant) and the other physical parameters in (A.1) are the same as in section 2.1 .

In the unforced case $\lambda=0$, equation (A.1) can describe small amplitude bending oscillations in a chain of coupled MEMS cantilevers, with coupling induced by an overhang [91]. The impulsive forcing term can be implemented 
using a feedback loop, see [92] and references therein. In the work [92], a nonlinear version of (A.1) was derived (for a single oscillator and $\zeta=0$ ) in order to model a MEMS resonator actuated with electrostatic pulses. In what follows, we show that (under a transversality condition) equation (A.1) is equivalent to the BK model with piecewise linear discontinuous friction law $F_{0}$ and pulling velocity $V=a-\zeta$ (equations (A.7)-(A.8) below).

The components $z_{n}$ correspond to continuous piecewise-differentiable functions, displaying jumps of derivatives at $t=t_{k}$. One obtains from (A.1)

$$
\left[\dot{z}_{n}\right]_{t_{k}}=\frac{\lambda}{m} \operatorname{sign}\left(\dot{z}_{n}\left(t_{k}^{-}\right)\right)
$$

with $[f]_{t_{0}}=f\left(t_{0}^{+}\right)-f\left(t_{0}^{-}\right)$denoting the jump discontinuity of a function $f$ at $t_{0}$. In order to map (A.1) to the piecewise linear BK model, we need the following technical lemma.

Lemma 1. Let u denote a continuous piecewise-differentiable function such that $u(0)=0, \dot{u}\left(0^{-}\right) \neq 0$. The following properties are equivalent:

$$
\begin{aligned}
\text { i) } & \operatorname{sign}\left([\dot{u}]_{0}\right)=\operatorname{sign}\left(\dot{u}\left(0^{-}\right)\right), \\
\text {ii) } & \operatorname{sign}\left([\dot{u}]_{0}\right)=[H(u)]_{0} .
\end{aligned}
$$

Proof. Let us first assume $i$ ) and show that ii) holds true. Since $\dot{u}\left(0^{+}\right)=\dot{u}\left(0^{-}\right)+$ $\left.[\dot{u}]_{0}, i\right)$ implies $\operatorname{sign}\left(\dot{u}\left(0^{+}\right)\right)=\operatorname{sign}\left(\dot{u}\left(0^{-}\right)\right)$. This equality implies $[H(u)]_{0}=$ $\operatorname{sign}\left(\dot{u}\left(0^{ \pm}\right)\right)$(since $\dot{u}\left(0^{ \pm}\right) \neq 0$ ), which leads to $i i$ ) using $i$ ).

Now let us show that ii) implies $i$ ). We prove this statement for $\dot{u}\left(0^{-}\right)>0$, the proof for $\dot{u}\left(0^{-}\right)<0$ being similar. The above assumptions imply $\operatorname{sign}\left([\dot{u}]_{0}\right)=$ $[H(u)]_{0} \geq 0$, hence $\dot{u}\left(0^{+}\right) \geq \dot{u}\left(0^{-}\right)>0$, leading to $[H(u)]_{0}=1$. From ii) we then get $\operatorname{sign}\left([\dot{u}]_{0}\right)=1$ and thus $\left.i\right)$ holds true.

In the sequel we restrict our attention to solutions of (A.1) satisfying the following transversality condition for all $n$ and all crossing times $t_{k}$ :

$$
\dot{z}_{n}\left(t_{k}^{-}\right) \neq 0 .
$$

Applying lemma 1 to $u(t)=z_{n}\left(t+t_{k}\right)-\zeta$, property $\left.i\right)$ is satisfied thanks to (A.2) (with $\lambda, m>0)$ and thus $\operatorname{sign}\left(\dot{u}\left(0^{-}\right)\right)=[H(u)]_{0}$, meaning that

$$
\operatorname{sign}\left(\dot{z}_{n}\left(t_{k}^{-}\right)\right)=\left[H\left(z_{n}-\zeta\right)\right]_{t_{k}} .
$$

Consequently, equation (A.1) and the transversality condition (A.3) lead to

$$
m \ddot{z}_{n}+\nu \dot{z}_{n}+k_{p} z_{n}=k_{c} \Delta_{d} z_{n}+\lambda \frac{d}{d t} H\left(z_{n}-\zeta\right) .
$$

Similarly, applying lemma 1-ii) to any solution of (A.5) satisfying (A.3), one establishes the equivalence between equations (A.1) and (A.5) under the above transversality condition. 
Assuming further $z_{n}(0) \neq \zeta$ for all $n$ and integrating (A.5) on $[0, t]$, one obtains the equivalent formulation

$$
m \dot{z}_{n}+\nu\left(z_{n}-\zeta-\frac{\lambda}{\nu} H\left(z_{n}-\zeta\right)\right)=\left(k_{c} \Delta_{d}-k_{p}\right) \int_{0}^{t} z_{n}(s) d s+p_{n}
$$

with

$$
p_{n}=m \dot{z}_{n}(0)+\nu\left(z_{n}(0)-\zeta\right)-\lambda H\left(z_{n}(0)-\zeta\right) .
$$

Let us either consider a finite lattice with periodic or free end boundary conditions, or bounded sequences $\left(z_{n}, \dot{z}_{n}\right)$ on an infinite lattice. In each case the linear map $k_{c} \Delta_{d}-k_{p}$ is invertible $\left(k_{c}, k_{p}>0\right)$ and one can introduce the new variable

$$
x_{n}(t)=\int_{0}^{t} z_{n}(s) d s+\left(k_{c} \Delta_{d}-k_{p}\right)^{-1} p_{n}-\frac{f_{0}}{k_{p}},
$$

where $f_{0}$ denotes an arbitrary constant. Substitution in (A.6) leads to the piecewise linear BK model

$$
m \ddot{x}_{n}+\tilde{F}\left(\dot{x}_{n}\right)+k_{p} x_{n}=k_{c} \Delta_{d} x_{n}
$$

where

$$
\tilde{F}(u)=\nu(u-\zeta)-\lambda H(u-\zeta)+f_{0} .
$$

This family of piecewise linear functions is parameterized by the slope $\nu$, the position $\zeta$ and the magnitude $\lambda$ of the jump discontinuity and the local maximum $f_{0}$. One recovers the piecewise linear BK model with pulling velocity $V$ and discontinuous friction force $f_{0} F_{0}\left(V+\dot{x}_{n}\right)=\tilde{F}\left(\dot{x}_{n}\right)$ (cf. equations (2) and (6)) by fixing $\zeta=a-V, f_{0}=\nu a$ and $\lambda=f_{0} \alpha$.

Acknowledgements. We thank the anonymous referees for providing valuable references and comments which have significantly improved the paper. We are also grateful to A. Vainchtein, J.-P. Françoise, M. Herrmann, B. Lombard and H. de Jong for helpful comments and stimulating discussions. G.J. would like to thank J. Schmittbuhl and R. Toussaint for stimulating his interest in the BK model several years ago.

[1] Y. Y. Kagan, Observational evidence for earthquakes as a nonlinear dynamic process, Physica D 77 (1994) 160-192.

[2] C. Scholz, The Mechanics of Earthquakes and Faulting, Cambridge University Press, 2002.

[3] B. Kaproth, C. Marone, Slow earthquakes, preseismic velocity changes, and the origin of slow frictional stick-slip, Science 341 (2013) 12291232.

[4] R. Burridge, L. Knopoff, Model and theoretical seismicity, Bull. Seismol. Soc. Am. 57 (1967) 341-371. 
[5] W. Huang, X. Wang, Biomimetic design of elastomer surface pattern for friction control under wet conditions, Bioinspiration and Biomimetics 8 (2013) 046001.

[6] J. M. Carlson, J. S. Langer, Properties of earthquakes generated by fault dynamics, Phys. Rev. Lett. 62 (1989) 2632.

[7] L. de Arcangelis, C. Godano, J. R. Grasso, E. Lippiello, Statistical physics approach to earthquake occurrence and forecasting, Physics Reports 628 (2016) 1-91.

[8] P. Moschetta, C. Mascia, Assessment of Predictor-Corrector strategy for the Burridge-Knopoff model, arXiv:1607.08267 [math.NA] (2016).

[9] J. S. Helman, W. Baltensperger, J. A. Holyst, Simple model for dry friction, Phys. Rev. B 49 (1994) 3831-3838.

[10] M. Weiss, F.-J. Elmer, Dry friction in the Frenkel-Kontorova-Tomlinson model: dynamical properties, Zeitschrift für Physik B Condensed Matter 104 (1) (1997) 55-69.

[11] A. Vanossi, N. Manini, M. Urbakh, S. Zapperi, E. Tosatti, Colloquium: Modeling friction: From nanoscale to mesoscale, Rev. Mod. Phys. 85 (2013) $529-552$.

[12] Y. Bar-Sinai, R. Spatschek, E. A. Brener, E. Bouchbinder, On the velocitystrengthening behaviour of dry friction, J. Geophys. Res. Solid Earth 119 (2014) 1738-1748.

[13] C. Marone, Laboratory-derived friction laws and their application to seismic faulting, Annual Review of Earth and Planetary Sciences 26 (1) (1998) 643-696.

[14] P. J. Blau, Friction Science and Technology: From Concepts to Applications, 2nd Edition, CRC Press, 2009.

[15] K. Chau, Landslides modeled as bifurcations of creeping slopes with nonlinear friction law, International Journal of Solids and Structures 32 (23) (1995) $3451-3464$.

[16] M. Di Bartolomeo, F. Massi, L. Baillet, A. Culla, A. Fregolent, Interplay between local frictional contact dynamics and global dynamics of a mechanical system, in: Gaëtan Kerschen (Ed.), Nonlinear Dynamics, Volume 1: Proceedings of the 33rd IMAC, A Conference and Exposition on Structural Dynamics, 2015, Springer International Publishing, Cham, 2016, pp. $1-10$.

[17] T. Putelat, J. Dawes, J. Willis, Regimes of frictional sliding of a springblock system, J. Mech. Phys. Sol. 58 (2010) 27-53. 
[18] B. Erickson, B. Birnir, D. Lavallée, Periodicity, chaos and localization in a Burridge-Knopoff model of an earthquake with rate-and-state friction, Geophys. J. Int. 187 (2011) 178-198.

[19] B. Erickson, B. Birnir, D. Lavallée, A model for aperiodicity in earthquakes, Nonlinear Processes in Geophysics 15 (2008) 1-12.

[20] F. Heslot, T. Baumberger, B. Perrin, C. Caroli, B. Caroli, Creep, stick-slip, and dry friction dynamics: Experiments and a heuristic model, Phys. Rev. E 49 (1994) 4973-4988.

[21] J. Wang, R. Hwang, One-dimensional dynamic simulations of slip complexity of earthquake faults, Earth Planets Space 53 (2001) 91-100.

[22] A. Ohmura, H. Kawamura, Rate- and state-dependent friction law and statistical properties of earthquakes, EPL (Europhysics Letters) 77 (2007) 69001 .

[23] M. Lebellego, Phénomènes ondulatoires dans un modèle discret de faille sismique, Ph.D. thesis, Université de Toulouse (2011).

[24] H. J. Melosh, Dynamical weakening of faults by acoustic fluidization, Nature 379 (6566) (1996) 601-606.

[25] F. Giacco, L. Saggese, L. de Arcangelis, E. Lippiello, M. Pica Ciamarra, Dynamic weakening by acoustic fluidization during stick-slip motion, Phys. Rev. Lett. 115 (2015) 128001.

[26] J. Wang, One-dimensional dynamical modelling of earthquakes: a review, Terr. Atmos. Ocean. Sci 19 (2008) 183-203.

[27] V. Acary, B. Brogliato, Numerical methods for nonsmooth dynamical systems. Applications in mechanics and electronics, Springer, 2008.

[28] J. Bastien, C. Lamarque, Theoretical study of a chain sliding on a fixed support, Mathematical Problems in Engineering 2009 (2009) 19 pages. doi: $10.1155 / 2009 / 361296$.

[29] X. Xiong, R. Kikuuwe, M. Yamamoto, Implicit Euler simulation of onedimensional Burridge-Knopoff model of earthquakes with set-valued friction laws, Advances in Computational Mathematics 41 (2015) 1039-1057.

[30] K. Vorvolakos, M. K. Chaudhury, The effects of molecular weight and temperature on the kinetic friction of silicone rubbers, Langmuir 19 (2003) $6778-6787$.

[31] J. P. Gong, Friction and lubrication of hydrogels - its richness and complexity., The Royal Society of Chemistry 20062 (2006) 544-552.

[32] J. M. Carlson, J. S. Langer, Mechanical model of an earthquake fault, Phys. Rev. A 40 (1989) 6470-6484. 
[33] J. Schmittbuhl, J. P. Vilotte, S. Roux, Propagative macrodislocation modes in an earthquake fault model, Europhys. Lett. 21 (1993) 375-380.

[34] S. Kostić, N. Vasović, I. Franović, K. Todorović, Dynamics of simple earthquake model with time delay and variation of friction strength, Nonlinear Processes in Geophysics 20 (5) (2013) 857-865.

[35] P. Español, Propagative slipping modes in a spring-block model, Phys. Rev. E 50 (1994) 227-235.

[36] J. Schmittbuhl, J. P. Vilotte, S. Roux, A dissipation-based analysis of an earthquake fault model, J. Geophys. Res. 101 (1996) 27741-27764.

[37] Y. Estrin, Y. Bréchet, On a model of frictional sliding, Pure Appl. Geophys. 147 (1996) 745-762.

[38] T. Shimamoto, Transition between frictional slip and ductile flow for halite shear zones at room temperature, Science 231 (1986) 711-714.

[39] F. Wu-Bavouzet, J. Clain-Burckbuchler, A. Buguin, P.-G. De Gennes, F. Brochard-Wyart, Stick-Slip: wet versus dry, The Journal of Adhesion 83 (2007) 761-784.

[40] R. FitzHugh, Impulses and physiological states in theoretical models of nerve membrane, Biophysical Journal 1 (1961) 445 - 466.

[41] E. C. Zeeman, Differential equations for the heartbeat and nerve impulse, in: Towards a theoretical biology, Vol. 4, Edinburgh University Press Edinburgh, 1972, pp. 8-67.

[42] M. Feingold, D. L. Gonzalez, O. Piro, H. Viturro, Phase locking, period doubling, and chaotic phenomena in externally driven excitable systems, Phys. Rev. A 37 (1988) 4060-4063.

[43] E. M. Izhikevich, Dynamical systems in neuroscience: The geometry of excitability and bursting, The MIT Press, 2007.

[44] J. H. E. Cartwright, E. Hernández-García, O. Piro, Burridge-Knopoff models as elastic excitable media, Phys. Rev. Lett. 79 (1997) 527-530.

[45] J. H. E. Cartwright, V. M. Eguíluz, E. Hernández-García, O. Piro, Dynamics of elastic excitable media, Int J. Bif. Chaos 9 (1999) 2197-2202.

[46] H. J. Xu, L. Knopoff, Periodicity and chaos in a one-dimensional dynamical model of earthquakes, Phys. Rev. E 50 (1994) 3577-3581.

[47] C. B. Muratov, Traveling wave solutions in the Burridge-Knopoff model, Phys. Rev. E 59 (1999) 3847-3857.

[48] E. Bouchbinder, E. A. Brener, I. Barel, M. Urbakh, Slow cracklike dynamics at the onset of frictional sliding, Phys. Rev. Lett. 107 (2011) 235501. 
[49] Y. Bar-Sinai, R. Spatschek, E. A. Brener, E. Bouchbinder, Instabilities at frictional interfaces: Creep patches, nucleation, and rupture fronts, Phys. Rev. E 88 (2013) 060403.

[50] Y. Bar-Sinai, R. Spatschek, E. A. Brener, E. Bouchbinder, Velocitystrengthening friction significantly affects interfacial dynamics, strength and dissipation, Sci. Rep. 5 (2015) 7841.

[51] J. C. Comte, P. Tchofo-Dinda, M. Remoissenet, Discrete Burridge-Knopoff model, with exact solitonic or compactlike traveling wave solution, Phys. Rev. E 65 (2002) 026615.

[52] J. E. Morales, G. James, A. Tonnelier, Traveling waves in a spring-block chain sliding down a slope, Phys. Rev. E 96 (2017) 012227.

[53] H. P. McKean Jr., Nagumo's equation, Adv. Math. 4 (1970) 209-223.

[54] J. Rinzel, J. B. Keller, Traveling wave solutions of a nerve conduction equation, Biophys. J. 13 (1973) 1313-1337.

[55] E. P. Zemskov, E. P. Zykov, V. S. Kassner, S. C. Muller, Stability of travelling fronts in a piecewise-linear reaction-diffusion system, Nonlinearity 13 (2000) 2063-2076.

[56] V. N. Biktashev, M. A. Tsyganov, Solitary waves in excitable systems with cross-diffusion, Proc. R. Soc. A 461 (2005) 3711-3730.

[57] V. Celli, N. Flytzanis, Motion of a screw dislocation in a crystal, J. Appl. Phys. 41 (1970) 4443-4447.

[58] N. Flytzanis, S. Crowley, V. Celli, High velocity dislocation motion and interatomic force law, J. Phys. Chem. Solids 38 (1977) 539-552.

[59] G. Fáth, Propagation failure of traveling waves in a discrete bistable medium, Physica D 116 (1998) 176-190.

[60] J. W. Cahn, J. Mallet-Paret, E. S. Van Vleck, Traveling wave solutions for systems of ODEs on a two-dimensional spatial lattice, SIAM J. Appl. Math. 59 (1998) 455-493.

[61] A. Tonnelier, McKean caricature of the FitzHugh-Nagumo model: traveling pulses in a discrete diffusive medium, Phys. Rev. E 67 (2003) 036105.

[62] C. E. Elmer, E. S. Van Vleck, Spatially discrete FitzHugh-Nagumo equations, SIAM J. Appl. Math. 65 (2005) 1153-1174.

[63] L. Truskinovsky, A. Vainchtein, Kinetics of martensitic phase transitions: Lattice model, SIAM J. Appl. Math. 66 (2005) 533-553.

[64] H. Schwetlick, J. Zimmer, Existence of dynamic phase transitions in a onedimensional lattice model with piecewise quadratic interaction potential, SIAM J. Math. Anal. 41 (2009) 1231-1271. 
[65] W. Atkinson, N. Cabrera, Motion of a Frenkel-Kontorova dislocation in a one-dimensional crystal, Phys. Rev. A 138 (1965) 763-766.

[66] O. Kresse, L. Truskinovsky, Mobility of lattice defects: discrete and continuum approaches, J. Mech. Phys. Solids 51 (2003) 1305-1332.

[67] A. Vainchtein, E. S. Van Vleck, Nucleation and propagation of phase mixtures in a bistable chain, Phys. Rev. B 79 (2009) 144-123.

[68] A. Vainchtein, E. S. Van Vleck, A. Zhang, Propagation of periodic patterns in a discrete system with competing interactions, SIAM J. Appl. Dyn. Systems 14 (2015) 523-555.

[69] H. J. Hupkes, B. Sandstede, Travelling pulses for the discrete FitzHughNagumo system, SIAM J. Appl. Dyn. Syst. 9 (2010) 827-882.

[70] Z. Zhi-fen, D. Tong-ren, H. Wen-zao, D. Zhen-xi, Qualitative theory of differential equations, Vol. 101 of Translations of Mathematical Monographs, American Mathematical Society, Providence, Rhode Island, 1992.

[71] J. Llibre, M. Ordóñez, E. Ponce, On the existence and uniqueness of limit cycles in planar continuous piecewise linear systems without symmetry, Nonlinear Anal. Real World Appl. 14 (2013) 2002-2012.

[72] A. C. Hindmarsh, ODEPACK, a systematized collection of ODE solvers, in: R. S. Stepleman (Ed.), Scientific Computing, Volume 1 of IMACS Transactions on Scientific Computation, North-Holland, Amsterdam, 1983, pp. $55-64$.

[73] J. P. Keener, Propagation and its failure in coupled systems of discrete excitable cells, SIAM J. Appl. Math. 47 (1987) 556-572.

[74] G. B. Ermentrout, D. H. Terman, Mathematical foundations of neuroscience, Springer, 2010.

[75] R. Piessens, E. de Doncker-Kapenga, C. Überhuber, D. Kahaner, QUADPACK. A Subroutine Package for Automatic Integration, Vol. 1 of Springer Series in Computational Mathematics, Springer-Verlag, Berlin Heidelberg, 1983.

[76] C. Kelley, Iterative Methods for Optimization, Frontiers in Applied Mathematics, Society for Industrial and Applied Mathematics, Philadelphia, 1999.

[77] M. Herrmann, K. Matthies, H. Schwetlick, J. Zimmer, Subsonic phase transition waves in bistable lattices models with small spinodal region, SIAM J. Math. Anal. 45 (2013) 2625-2645.

[78] Y. Earmme, J. Weiner, Dislocation dynamics in the modified FrenkelKontorova model, J. Appl. Phys. 48 (1977) 3317-3331. 
[79] O. Kresse, L. Truskinovsky, Lattice friction for crystalline defects: From dislocations to cracks, J. Mech. Phys. Solids 52 (2004) 2521-2543.

[80] L. I. Slepyan, A. Cherkaev, E. Cherkaev, Transition waves in bistable structures. II. Analytical solution: wave speed and energy dissipation, J. Mech. Phys. Solids 53 (2005) 407-436.

[81] A. Vainchtein, The role of spinodal region in the kinetics of lattice phase transitions, J. Mech. Phys. Solids 58 (2010) 227-240.

[82] L. Truskinovsky, A. Vainchtein, Solitary waves in a nonintegrable FermiPasta-Ulam chain, Phys. Rev. E 90 (2014) 42903.

[83] M. A. Collins, A quasicontinuum approximation for solitons in an atomic chain, Chem. Phys. Lett. 77 (1981) 342-347.

[84] P. Rosenau, Dynamics of nonlinear mass-spring chains near the continuum limit, Phys. Lett. A 118 (1986) 222-227.

[85] M. Argentina, P. Coullet, V. Krinsky, Head-on collisions of waves in an excitable FitzHugh-Nagumo system: a transition from wave annihilation to classical wave behavior, J. theor. Biol. 205 (2000) 47-52.

[86] M. A. Tsyganov, V. N. Biktashev, J. Brindley, A. V. Holden, G. R. Ivanitsky, Waves in systems with cross-diffusion as a new class of nonlinear waves, Physics-Uspekhi 50 (2007) 263-286.

[87] M. A. Tsyganov, V. N. Biktashev, Classification of wave regimes in excitable systems with linear cross-diffusion, Phys. Rev. E 90 (2014) 062912.

[88] N. A. Venturi, F. Nori, Marginal stability and chaos in coupled faults modelled by nonlinear circuits, Phys. Rev. Lett. 74 (1995) 74-77.

[89] J. E. Morales Morales, Ondes localisées dans des systèmes mécaniques discrets excitables, Ph.D. thesis, Université de Grenoble (2016).

[90] R. A. Harris, Large earthquakes and creeping faults, Reviews of Geophysics 55 (1) (2017) 169-198.

[91] M. Sato, B. E. Hubbard, L. Q. English, A. J. Sievers, B. Ilic, D. A. Czaplewski, H. G. Craighead, Study of intrinsic localized vibrational modes in micromechanical oscillator arrays, Chaos: An Interdisciplinary Journal of Nonlinear Science 13 (2) (2003) 702-715.

[92] J. Juillard, A. Bonnoit, E. Avignon, S. Hentz, E. Colinet, Large amplitude dynamics of micro-/nanomechanical resonators actuated with electrostatic pulses, Journal of Applied Physics 107 (1) (2010) 014907. 